\title{
Apocalipsis cum figuris
}

Die Analyse von Leverkühns fiktiven Kompositionen und ihren intermedialen Transpositionen wird in diesem Kapitel mit der Apocalipsis cum figuris fortgesetzt. Das im Jahr 1919 geschriebene Oratorium wird im 34. Kapitel des Romans Doktor Faustus geschildert: Der Titel des fiktiven Werkes lässt es sofort in eine Tradition künstlerischer Werke einschreiben, die sich u. a. von der Offenbarung des Johannes inspirieren lassen. So Rita Müller-Fieberg über die Faszination vieler Künstler*innen für den biblischen Text: ${ }^{1}$

Zum einen stellt die Offenbarung des Johannes in ihrer kunstvollen ästhetischen Formung selbst ein poetisches Meisterwerk dar und vermochte daher auch immer wieder andere Autoren dazu einladen, aus ihrer Sprache, aus ihren Bildern und ihrer Dramatik zu schöpfen.

Der erste Teil des vorliegenden Kapitels setzt sich mit einer Definition von Apokalypse und mit Typen von Apokalypsen auseinander, die dann auf Manns Roman übertragen werden. Als wichtiges Element eines apokalyptischen Diskurses profiliert sich das Zusammenspiel von Immanenz und Transzendenz: In Doktor Faustus wird dieser Diskurs durch das Oratorium Leverkühns musikalisch und durch die Vorstellung der Ideen eines Münchener Kreises politisch dekliniert. Sowohl auf der musikalischen als auch auf der politischen Ebene bewegt sich das 34. Kapitel von Doktor Faustus noch auf einem ersten immanenten Zustand. Die ursprüngliche, nicht ausschließlich negativ konnotierte Bedeutung des Wortes Apokalypse und des Wortes Katastrophe spielen aufgrund der von Leverkühn verwendeten mittelalterlichen Quellen für die Komposition und der Ausbildung

\footnotetext{
${ }^{1}$ Müller-Fieberg, Rita: Das „,neue Jerusalem“ - Vision für alle Herzen und alle Zeiten? Eine Auslegung von Offb 21,1-22,5 im Kontext von alttestamentlich-frühjüdischer Tradition und literarischer Rezeption. Berlin (u. a.): Philo 2003, S. 28 f.
} 
des Erzählers eine zentrale Rolle; zugleich stützt sich dieser Teil u. a. auf Derridas Schrift, die ein historisch passendes Pendant zum zweiten Teil des Kapitels bietet. So wie Derrida in den 1980er Jahren den apokalyptischen Diskurs rezipiert und auszuloten versucht, so setzen sich die drei behandelten Kompositionen jeweils von Konrad Boehmer, Karl-Wieland Kurz und Humphrey Searle zwischen 1980 und 1988 nicht nur mit Leverkühns Werk, sondern auch mit dessen apokalyptischem Diskurs auseinander. Dieser Aspekt lässt sich als transmedial einstufen: Die Apokalypse ist an keine „bestimmte mediale Präsentationsform gebunden", 2 dementsprechend werden in diesem Kapitel medienübergreifende Rekursverfahren auf den apokalyptischen Diskurs untersucht.

Boehmers Apocalipsis cum figuris zeichnet sich durch die Kombination von Musikstilen und Musikgattungen aus und schafft durch das Ende mit einem Leopardi-Zitat und dem Schrei eines neugeborenen Kindes den Sprung zu einer keineswegs einbahnig interpretierbaren Transzendenz. Sowohl das Werk von Boehmer als auch das von Kurz besitzen einen enzyklopädischen Charakter und üben, intermedial betrachtet, einen ergänzenden Effekt aus, da sie über die im Roman erwähnten apokalyptischen Quellen hinausgehen und noch weitere verwenden, z. B. die Sybillinischen Weissagungen und kasuistische Texte. Zugleich distanziert sich die Komposition von Kurz bereits im Titel von der Vorlage: Es handelt sich in diesem Fall um eine konturlose Apokalypse sine figuris, in der nicht Bildhaftigkeit, sondern gerade ihr Gegenteil als formbildend konzipiert wird. Das sine figuris und das Werk selbst lassen sich allerdings nicht nur als Reaktion eines sekundären intermedialen Produktes auf das 34. Kapitel von Thomas Manns Doktor Faustus, sondern (sowohl in Anbetracht der Entstehungszeit als auch der expliziten Erwähnung von Boehmers Doktorarbeit) als Reaktion eines sekundären intramedialen Produktes auf Boehmers Apocalipsis betrachten. Während die Kompositionen von Boehmer und Kurz nicht das Ziel verfolgen, den Klang von Leverkühns Oratorium zu rekonstruieren, sondern eher dessen Radikalität ihrer musikalischen Epoche anzupassen, versucht die Komposition von Searle dagegen, sich so präzise wie möglich an der Vorlage zu orientieren. Daraus resultiert eine Reflexion über Mediendifferenzen und die Rezeption von intermedialen literarischen Werken: Die Uneindeutigkeit des Textes bezüglich des musikalischen Stils wird etwa im Medium der Musik durch einen präzisen Einsatz „,der Methode der Komposition mit zwölf nur aufeinander bezogenen Tönen" ${ }^{* 3}$ gelöst. Alle drei Kompositionen lassen sich als Medienkombinationen definieren, die mindestens über

\footnotetext{
${ }^{2}$ Rajewsky: Intermedialität, S. 73. Siehe auch Wolfs Definition von ,transmedial ‘ als „media-neutral“. Wolf: Narrativity in Instrumental Music?, S. 480.

${ }^{3}$ Schönberg: Komposition mit zwölf Tönen, ebd., Herv. i. O.
} 
die Medien Musik und Text verfügen, im Fall von Kurz auch über das Medium Kunst: Der intermediale Bezug in der Vorlage wird somit zu einem materiell präsenten Medium im sekundären intermedialen Produkt.

\title{
4.1 Apokalypsen in Doktor Faustus
}

Eine Ausführung über Typen von Apokalypsen im Text und in den Vertonungen, apokalyptische Motive und Apokalyptiker*innen muss von einer Definition von Apokalypse ausgehen. In seiner Schrift von 1983 mit dem Titel Von einem neuerdings erhobenen apokalyptischen Ton in der Philosophie erläutert Jacques Derrida die Etymologie des Wortes, um zu einer Definition zu gelangen: ${ }^{4}$

\begin{abstract}
Apokalypto war sicherlich ein gutes Wort für gala'. Apokalypto, ich entdecke [decouvre], ich enthülle [dévoile], ich offenbare [révèle] die Sache, die ein Körperteil, der Kopf oder die Augen, sein kann, ein geheimer Teil, das Geschlecht, oder was auch immer da verborgen zu halten ist, ein Geheimnis, die zu verbergende Sache, eine Sache, die weder gezeigt noch gesagt, die vielleicht bedeutet wird, aber zunächst nicht dem Augenschein preisgegeben werden kann oder darf. Apokekalymmenoi logoi, das sind anstößige Reden. Es geht also um das Geheimnis und die pudenda.
\end{abstract}

Bei einer Apokalypse handelt es sich also um die Offenbarung von Geheimnissen und Verborgenem, um etwas, das man nicht gerne hören möchte und was sich mit Schamgefühlen, Sexualität und Tabu-Themen verbinden kann. Dem Zitat lässt sich bereits entnehmen, worauf Jürgen Brokoff explizit hinweist: Der Fokus einer Apokalypse liegt vielmehr auf dem ,,Vorgang des Offenbarens, Enthüllens, Aufdeckens" ${ }^{\text {5 }}$ als auf dem Zustand des Offenbarten, Enthüllten und Aufgedeckten. Im Vordergrund steht also die Performanz der apokalyptischen Verkündung, die Brokoff „ohne das Medium Sprache nur schwer vorstellbar erscheint“ 6 Diese Notwendigkeit eines Rückgriffs auf das sprachliche Medium wird sowohl durch den Bezug auf Dürers Holzschnitte in Leverkühns Oratorium als auch durch die Kompositionen des zweiten Teils dieses Kapitels zugleich widerlegt und bestätigt: Die vorher genannten Beispiele bedienen sich doch anderer Medien, basieren aber gleichwohl auf einer sprachlichen Vorlage. Leverkühns Komposition, die auch in

\footnotetext{
${ }^{4}$ Derrida: Apokalypse, S. 12, Anmerkungen des Übersetzers, Herv. i. O.

${ }^{5}$ Brokoff, Jürgen: Die Apokalypse in der Weimarer Republik. München: Fink 2001, S. 7. ${ }^{6}$ Ebd.
} 
ihrer Fiktionalität immerhin auf eine fiktive Aufführung ausgerichtet ist, unterstreicht sowohl die Bildhaftigkeit des apokalyptischen Textes durch die Ergänzung cum figuris als auch durch den Rekurs auf das Medium Musik die Performanz und Prozesshaftigkeit des apokalyptischen Offenbarens.

Apokalypse und Weltuntergang werden nicht selten - und das nicht nur in der Alltagssprache - gleichgesetzt. Brokoffs Untersuchung zufolge lässt sich diese Verwechslung darauf zurückführen, dass die Offenbarung des Johannes, die den Weltuntergang schildert, die letzte Schrift des Neuen Testaments darstellt. Übersehen werde aber, dass ,,[d]er Weltuntergang [...] keineswegs den Schlußpunkt der Johanneischen Erzählung " ${ }^{\text {7 }}$ bilde; ihm folge ,,,das neue Jerusalem“, das immerwährende Reich Gottes“. ${ }^{8}$ Auf dieses strukturelle Zusammenspiel von Immanenz und Transzendenz legt Brokoffs Studie, die beabsichtigt, die Apokalypse aus literaturwissenschaftlicher Perspektive, also die Apokalypse als Text zu erforschen, sehr viel Wert: Die immanente Welt wird mit dem Ziel vernichtet, die „Errichtung

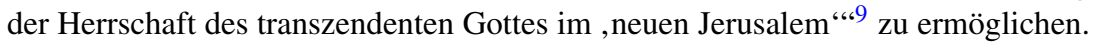
Die Apokalypse als Redeform ist daher eine Rede von Untergang und Neubeginn; infolgedessen bedarf sie mindestens einer zweimotivischen Struktur, wobei das zweite Motiv, das Motiv des Neubeginns - wie in der Offenbarung - lediglich angedeutet werden kann und folglich oft übersehen wird.

Derrida sagt in der bereits erwähnten Schrift Folgendes: ${ }^{10}$

Man weiß nie (weil es nicht mehr der Ordnung des Wissens unterliegt), wem die apokalyptische Sendung zukommt, sie springt von einem Sende-Ort zum anderen (und ein Ort wird immer im Ausgang [à partir] vom mutmaßlichen Senden bestimmt), sie geht von einer Bestimmung, von einem Namen und einem Ton zum anderen, sie verweist immer auf den Namen und den Ton des anderen, der da ist, aber nur als derjenige, der da gewesen ist und noch kommen muß, der in der Gegenwart der Erzählung nicht mehr da oder noch nicht da ist.

Auch im vorliegenden Kapitel wird versucht, die Logik der zahlreichen intraund intermedialen Verweise des apokalyptischen Diskurses, die in Leverkühns Oratorium und in den Kompositionen von Boehmer, Kurz und Searle enthalten sind, offenzulegen. Diese verschiedenen, aber aufeinander bezogenen

\footnotetext{
${ }^{7}$ Ebd.

${ }^{8}$ Ebd.

${ }^{9}$ Ebd., S. 10.

${ }^{10}$ Derrida: Apokalypse, S. 71, Anmerkungen des Übersetzers.
} 
Deklinationen des apokalyptischen Diskurses werden definiert und in Verbindung sowohl mit kulturwissenschaftlichen Studien zur Apokalypse als auch mit Intermedialitätstheorien gebracht.

\subsubsection{Die musikalische Apokalypse: Adrian Leverkühns Oratorium}

Im vorliegenden Abschnitt wird zunächst einmal auf die musikalische Deklination des apokalyptischen Diskurses in Doktor Faustus und dementsprechend auf die Darstellung von Leverkühns Oratorium durch die Erzählinstanz Zeitblom eingegangen. Dem Titel des fiktiven Werkes lässt sich entnehmen, dass Adrian Leverkühn nicht nur aus der Sprache und der Dramatik der Offenbarung schöpft, sondern auch aus den Bildern, was durch die Spezifizierung cum figuris deutlich zum Ausdruck kommt. Diese Spezifizierung verweist zudem auf eine weitere Vorlage der Komposition: Dürers Druckwerk gleichnamigen Titels. Die dynamische Verflechtung dreier Medien (Text, Musik und Kunst) wird im fiktiven paratextuellen Hinweis bereits programmatisch deklariert: Leverkühns Oratorium ist das fiktive Produkt zahlreicher intermedialer Bezüge und Medientransformationen. Denn weder die Offenbarung des Johannes noch Dürers Holzschnitte stellen die einzigen Quellen von Leverkühns Apocalipsis und des 34. Kapitels dar: Das Oratorium wird als „Resumé aller Verkündigungen des Endes“ (DF: 520) bezeichnet und so könnten ebenfalls alle Kompositionen bezeichnet werden, die Eingang in den zweiten Teil des vorliegenden Kapitels finden.

Diese „Vermischung der Stimmen, Gattungen und Codes“11 in Leverkühns Komposition und in den später betrachteten Musikwerken ist laut Derrida dem apokalyptischen Ton angeboren. Selbst der Sprache der Offenbarung des Johannes wird nicht nur von Müller-Fieberg Bildhaftigkeit zugesprochen, sondern auch Albrecht Dürer transferierte und transformierte eine bereits vorhandene Qualität des biblischen Textes in das Medium der Kunst und Adrian Leverkühn setzt sich als Ziel, eine bildhafte Komposition zu schreiben, die Bezug auf den apokalyptischen Diskurs nimmt. ${ }^{12}$

Das sogenannte ,apokalyptische“ Kapitel von Thomas Manns Doktor Faustus, also das 34. Kapitel, weist eine dreiteilige Struktur auf und entspricht daher Brokoffs vorher präsentierter Definition der Apokalypse als Redeform, die eine mindestens zweimotivische Struktur besitzt. Das 34. Kapitel konzentriert sich

\footnotetext{
${ }^{11}$ Derrida: Apokalypse, S. 76.

${ }^{12}$ Siehe auch Brokoff: Die Apokalypse in der Weimarer Republik, S. 7.
} 
im ersten Teil vor allem auf Adrian Leverkühn und seine Vorarbeiten an der Komposition Apocalipsis cum figuris, sprich: auf seine Lektüre zahlreicher apokalyptischer Texte der Antike und des Mittelalters. Bereits hier kommen das historische Endzeitgefühl und verschiedene Aspekte von Leverkühns Komposition zur Sprache, so etwa die Präsenz eines Erzählers, der durch die chiastisch organisierten Worte „Das Ende kommt, es kommt das Ende“ (DF: 519) den Weltuntergang verkündet und dann diese Botschaft einem Responsorium abgibt, das sie „unvergeßlich wiederholt“ (ebd.) und sich aus ,Zwei vierstimmigen, gegeneinander bewegten Chören“ (ebd.) zusammensetzt. Neben der Beobachtung, dass auch das Responsorium die Zweiteiligkeit der Apokalypse reproduziert, ist es hier auffällig, dass nicht nur formale und inhaltliche Besonderheiten von Leverkühns Oratorium, sondern auch harmonische geschildert werden. So werden beispielsweise musikalisch die Worte des Erzählers ,,in einer geisterhaften, auf liegenden Fremd-Harmonien ruhenden, aus reinen Quarten- und verminderten Quintenschritten gefügten Melodik“ (ebd.) wiedergegeben. Zeitbloms Beschreibung von Leverkühns Oratorium fällt nicht nur unter den Modus des intermedialen telling, sondern auch des intermedialen showing, da diese sehr detaillierte Beschreibung des Werkes zugleich auch auf die „Vergegenwärtigung eines (fiktiven) Klangeindrucks"13 zielt und sich daher sowohl als evozierend als auch als simulierend einstufen lässt. ${ }^{14}$

Der zweite Teil des Kapitels, in Klammern „Fortsetzung“ (DF: 525) genannt, befasst sich mit den Tafelrunden des Kridwiß-Kreises, also mit den Treffen einer Gruppe von Intellektuellen in München, die durch den Rückgriff auf Mythen und das Ziel einer Vereinfachung der Wissenschaft den ideologischen Boden für die spätere politische Situation in Deutschland bereiten. Der dritte Teil, in Klammern „Schluß“ (DF: 538) genannt, geht noch einmal auf Leverkühns Werk ein und besteht hauptsächlich aus Zeitbloms Bewertung der Komposition. In ihr werden Stil, Mittel und einige Merkmale wieder durch ein vorherrschendes telling mit fließenden Grenzen zum showing weiter beschrieben.

Wie durch die Explikation der drei Teile ersichtlich wurde, besitzt das Kapitel eine A-B-A-Struktur, die sich aus einer Präsentation des Hauptthemas, einer Fortsetzung und seiner Variation sowie aus einem Schluss, der das Hauptthema erneut aufgreift und vertieft, zusammensetzt. Formal betrachtet, gibt es viele musikalische Vorlagen, die einer solchen Struktur zugrunde liegen und die

\footnotetext{
${ }^{13}$ Gess u. Honold: Einleitung, S. 8.

${ }^{14} \mathrm{Zu}$ den Begriffen vgl. 1.1.5.
} 


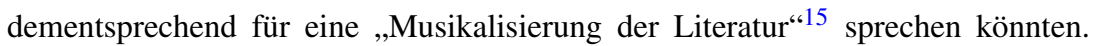
Strukturell betrachtet ist es nicht so, dass das Kapitel selbst versucht, die zweiteilige Struktur der Apokalypse zu reproduzieren. Infolgedessen könnte man der in einem Sammelband zur Aktualität des Apokalyptischen präsentierten Auffassung von Briese, Faber und Podewski zustimmen, dass dort ,[w]o Apokalyptisches drauf steht, [...] Apokalyptisches gerade nicht drin“" ist. ${ }^{16}$ Auch aus inhaltlicher Sicht bewegt sich das Kapitel auf dem immanenten Stadium des ,krisenhaften Zustand[s] A", 17 für den kein Neubeginn vorgeschlagen wird. Was durch die Struktur des Kapitels zum Tragen kommt, ist ein apokalyptischer Diskurs, an dessen Anfang und Ende die Musik steht. Folglich wäre es auch nicht unangebracht, von einer $\mathrm{A}^{1}-\mathrm{A}^{2}-\mathrm{A}^{1}$-Struktur $\mathrm{zu}$ sprechen, denn der zentrale Teil des Kapitels könnte als politische Modulation eines vorrangig musikalischen Themas interpretiert werden. Zweifellos werden durch Zeitbloms Erzählen im 34. Kapitel sowie in weiteren Kapiteln des Romans verschiedene Deklinationen des apokalyptischen Diskurses sichtbar; im Folgenden wird aufgrund der Akzentuierung der vorliegenden Studie vor allem auf die musikalische Ebene eingegangen, ohne die politische - die durch den Kridwiß-Kreis zum Ausdruck kommt - zu vernachlässigen.

Adrian Leverkühns Oratorium schafft noch nicht den Sprung zur Transzendenz und zur Erlösung. Was die Komposition widerspiegelt, ist ein äußerst produktiver Krisenzustand der Musik, die sich neu erfinden möchte und zu diesem Zweck disparate Musikstile und -mittel anwendet. Eine große Inspirationsquelle des Werkes stellt das Mittelalter und die Renaissance dar, sowohl in der Auswahl der apokalyptischen Texte und Kunstwerke, die zu den fiktiven Prätexten zählen, als auch in der musikalischen Faktur selbst. Leverkühn stützt sich beispielsweise auf Dante, besonders auf sein Inferno, auf , die ekstatischen Erlebnisse der Mechthild von Magdeburg“ (DF: 517) und der Hildegard von Bingen, auf die Historia ecclesiastica gentis Anglorum von Beda Venerabilis und auf vergleichbare Texte italienischen Ursprungs, wie beispielsweise die ,Dialoge[] Gregors des päpstlichen Sangesmeisters“ und die „Vision Alberichs, des Mönchs von Monte

\footnotetext{
${ }^{15}$ Rajewsky: Intermedialität, S. 7. Vgl. auch Werner Wolfs Einschränkung des Begriffs: Wolf, „The musicalization of fiction“, S. 134 (siehe auch Rajewsky: Intermedialität, S. 39 f.)

${ }^{16}$ Briese, Olaf, Richard Faber u. Madleen Podewski: Einleitung. In: Dies. (Hrsg.): Aktualität des Apokalyptischen. Zwischen Kulturkritik und Kulturversprechen. Würzburg: Königshausen \& Neumann 2015, S. 7-37, hier: S. 18.

${ }^{17}$ Ebd., S. 11.
} 
Cassino“, ${ }^{18}$ also allgemein gefasst verwendet er „früh-christliche[] und mittelalterliche[] Visionsliteratur und Jenseitsspekulation“ (DF: 518). Zeitblom liefert Hinweise zum Verfahren, dem Leverkühn folgt, um diese Texte zu kombinieren: Leverkühn sammle ,alle ihre Elemente in einem Brennpunkt“ (DF: 518), fasse „sie in später künstlerischer Synthese“ (DF: 518 f.) zusammen, um dann den Rezipient*innen ,nach unerbittlichem Auftrag der Menschheit den Spiegel der Offenbarung vor Augen“ (DF: 519) zu halten, ,damit sie darin erblicke, was nahe heran[...]komm[t]“ (ebd.). Es handelt sich nach Zeitbloms Wiedergabe dabei um ein Verfahren, das mit einer moralischen Absicht auf eine Synthese vieler Prätexte abzielt.

Auch in der musikalischen Faktur des Werkes sind Rückgriffe auf Formen, die vor der Barockzeit zu finden waren, enthalten: Dazu zählt etwa die „Chorfuge zu den Worten des Jeremias“ (DF: 523), die sich ,,auf die archaische Fugenform gewisser Canzonen und Ricercaren der Vor-Bach'schen Zeit" (DF: 524) stützt, ,in denen das Fugenthema nicht immer eindeutig definiert und festgehalten ist“ (ebd.). Gleichzeitig handelt es sich aber um eine Komposition, die verschiedene musikalische Stile parodiert: den französischen Impressionismus, die „bürgerliche Salonmusik, Tschaikowsky, Music Hall“ (DF: 545) und Jazz. ${ }^{19}$ Adrian Leverkühn experimentiert mit Rhythmen, Stilen und Formen und beschäftigt sich sogar mit der Frage nach der Denaturierung des Klanges, die Zeitblom als „die früheste Errungenschaft der Tonkunst" (DF: 542) definiert und die immer noch ein zentrales Anliegen von Komponist*innen der Nachkriegszeit, etwa von Konrad Boehmer, darstellt. ${ }^{20}$ Neues und Altes greifen ineinander; die Komposition lässt sich jedoch nicht als dodekaphonisch klassifizieren: Ein Neubeginn, also eine Überwindung der musikalischen Immanenz durch ein neues kompositorisches System, steht nicht hier, sondern erst in der Weheklag im Vordergrund.

Der Übergang zum Zustand B ist also nicht in diesem Kapitel zu suchen: Die musikalische Apokalypse setzt fort und schafft den Sprung zu einer musikalischen

\footnotetext{
${ }^{18}$ Die letzten beiden Quellen sind nur in der Taschenbuchausgabe zu finden. Siehe Mann, T.: Doktor Faustus. Das Leben des deutschen Tonsetzers Adrian Leverkühn, erzählt von einem Freunde. Frankfurt am Main: Fischer 2013, 39. Aufl., S. 474.

${ }^{19}$ Bemerkenswert ist, dass diese „Parodie verschiedenster musikalischer Stile“ (DF: 545) mit der „Grundsprache des Haupt-Orchesters“ (ebd.) kombiniert wird, was sich mit Hagens To Zeitblom und der Kombination der Hardangerfiedel mit einem klassischen Orchester vergleichen lässt. Vgl. 7.2.2. Die Rolle der Parodie in Thomas Manns Werken und in Doktor Faustus wurde von vielen Literaturwissenschaftler*innen untersucht, z. B. von Steen, Inken: Parodie und parodistische Schreibweise in Thomas Manns „Doktor Faustus“. Tübingen: Max Niemeyer 2001. Vgl. auch Ent: 45: „Ich kenne im Stilistischen eigentlich nur noch die Parodie“.
}

${ }^{20}$ Vgl. 4.2.1. 
Transzendenz erst mit der Anwendung des strengen Satzes und der Komposition der Dr. Fausti Weheklag. Betrachtet man die verschiedenen Typen von Apokalypsen, die in dem bereits erwähnten Sammelband von Briese, Faber und Podewski vorgestellt werden, so könnte sich in Doktor Faustus eine ,[i]nverse Warnapokalypse“ ${ }^{\text {21 }}$ profilieren. Im Roman droht zwar ,[e]in unerwünschtes, vernichtendes Ereignis [...] unweigerlich einzutreten“",22 nämlich die komplette Sterilität der Kunst, dieses Ereignis wird jedoch ,durch einen grundlegenden Gesinnungs- und Handlungswandel in der Gegenwart abgewendet" ${ }^{23}$ und zwar durch die Anwendung eines kompositorischen Systems, das Adrian Leverkühn schon seit langer Zeit vorschwebte. Berechtigt wäre aber der Einwand, dass nicht nur von einem politischen Gesichtspunkt aus, sondern auch in Sachen Musik keine echte Erlösung stattfindet: Im Endeffekt ist nur das ,hohe g eines Cello“ (DF: 711) am Ende der Weheklag in der Lage, nach den Worten des Erzählers den Sinn zu wandeln und eine metaphorische Assoziation zu einem „Licht in der Nacht“ (ebd.) zu ermöglichen. Hätte man dementsprechend Schwierigkeiten, eine musikalische Erlösung durch die Dodekaphonie im Roman zu sehen, so wäre es angebracht, von einer „,[e]motional-wunschhafte[n] Verneinungs- und Untergangsapokalypse“ ${ }^{\text {24 }}$ zu sprechen. Die bereits erwähnten Autor*innen des Sammelbandes definieren diese als eine moderne Art von Apokalypse, deren Entstehung sich im 19. Jahrhundert zeitlich situieren lässt: Diese Apokalypsen sind „um jeden zweiten Teil, den der Erlösung, beschnitten“ 25 oder begreifen „,die Erlösung gerade als Übergang in ein non-humanes Nichts“. ${ }^{26}$ Die Möglichkeit einer Erlösung der Musik durch ihren Neubeginn von einem einzigen Ton, von jenem g des Cello, könnte für zu schwach gehalten werden: Folglich wäre keine Erlösung vorhanden. Es könnte ebenfalls argumentiert werden, dass auch hier die Erlösung als Übergang in ein menschlich unbegreifliches Nichts zu begreifen ist, was jedoch Brokoff zufolge immerhin die Präsenz eines zweiten transzendentalen Stadiums und infolgedessen die mindestens zweimotivische Struktur der Apokalypse bestätigt.

Nachdem die Art des musikbezogenen apokalyptischen Diskurses in Doktor Faustus definiert wurde, sei nun auf einige Elemente eingegangen, die charakteristisch für apokalyptische Diskurse und Darstellungen sind und die man in Zeitbloms Beschreibung von Leverkühns Komposition wiederfindet. Die Wahl

\footnotetext{
${ }^{21}$ Briese, Faber u. Podewski: Aktualität des Apokalyptischen, S. 21.

${ }^{22}$ Ebd.

${ }^{23}$ Ebd., Herv. i. O.

${ }^{24}$ Ebd., S. 22.

${ }^{25}$ Ebd.

${ }^{26}$ Ebd.
} 
der Figuren und Inhalte verdeutlicht eine Interaktion zwischen Immanenz und Transzendenz, denn der Zeuge der Apokalypse Leverkühns warnt vor der bevorstehenden Katastrophe, indem er vom Jüngsten Gericht und von der Hölle berichtet. Der Chor und das Orchester unterstützen sein Erzählen beispielsweise durch Geheule, das als Thema fungiert, durch zahlreiche Arten von Glissandi, die auch auf die menschliche Stimme angewandt werden ${ }^{27}$ und durch die Emulation eines „Pandämonium des Lachens“ (DF: 548) im letzten Teil der Komposition: Es wird also versucht, Klänge und Geräusche der Hölle zu reproduzieren. Eine Kritik und Umwertung von Kompositionstendenzen, die den Weg für die streng zwölftönige Weheklag bereitet, ist wesentlicher Bestandteil des musikalischen Stückes, denn dieses sei

von dem Paradoxon beherrscht (wenn es ein Paradoxon ist), ${ }^{28}$ daß die Dissonanz darin für den Ausdruck alles Hohen, Ernsten, Frommen, Geistigen steht, während das Harmonische und Tonale der Welt der Hölle, in diesem Zusammenhang also einer Welt der Banalität und des Gemeinplatzes, vorbehalten ist. (DF: 544)

Auf die Struktur des Jenseits wird im Oratorium auch mittels der Interaktion des Tonalen und des Dissonanten angespielt, die der Interaktion zwischen Immanenz und Transzendenz gegenübergestellt werden kann. Dass es sich aber lediglich um einen Versuch der immanenten Welt handelt, das transzendente Jenseits abzubilden, was im Zentrum der meisten apokalyptischen Verkündungen steht, scheint von der Behandlung und Konzeption des vokalen und orchestralen Parts bestätigt. So Zeitblom:

Chor und Orchester stehen einander nicht als das Menschliche und das Dingliche klar gegenüber; sie sind ineinander aufgelöst: der Chor ist instrumentalisiert, das Orchester vokalisiert, - in dem Grade und zu dem Ende, daß tatsächlich die Grenze zwischen Mensch und Ding verrückt erscheint [...]. (DF: 544)

Chor und Orchester verkörpern dieser Beschreibung entsprechend immer noch die materielle Welt auf der Suche nach einer neuen musikalischen Sprache, die aber immerhin vom Status quo aus auf diese einzutretende musikalische Phase blickt und über die Möglichkeiten ihrer Umsetzung durch vielfältiges Experimentieren, intensives Interagieren und die Aufhebung von Trennungen reflektiert.

\footnotetext{
${ }^{27}$ Vgl. DF: 543.

${ }^{28}$ Der Einschub in Klammern könnte als ein weiteres Indiz für die Unzuverlässigkeit des Erzählers interpretiert werden. Zur Unzuverlässigkeit Zeitbloms vgl. Kap. 7. Des Weiteren weist Börnchen darauf hin, dass sich Thomas Manns Roman ,gerade in seinem Hang zum exzessiven Selbstkommentar selbst dekonstruiert“. Börnchen: Kryptenhall, S. 68.
} 
Dieses Schauen bildet den Kern der Apokalypse, die Derrida als „Kontemplation [...] oder eine Inspiration [...] der Schau“ ${ }^{29}$ definiert. Wichtiger Schritt zur Erreichung des Zustands B ist außerdem die „Vernichtung der Differenz selbst“, ${ }^{30}$ was sich durch die Vernichtung der weltlichen, in diesem Fall musikalischen, Sprache umsetzen lässt. ${ }^{31}$

Eine Apokalypse bedarf eines Apokalyptikers: Im Rahmen der Komposition selbst übernimmt der Zeuge diese Funktion und im Rahmen der intradiegetischen Dimension des Romans spielt Adrian Leverkühn diese Rolle. Umberto Eco beispielsweise grenzt in Bezug auf die Massenkultur Apokalyptiker*innen von Integrierten $a b:^{32}$

\begin{abstract}
Während die Apokalyptiker gerade dadurch überleben, daß sie Theorien über den Zerfall ausbilden, versagen sich die Integrierten weitgehend der Theoriearbeit; sie erzeugen und übermitteln ihre Botschaften in unbefangener Leichtigkeit, tagtäglich, auf allen Ebenen. Die Apokalypse ist eine Besessenheit des dissenters, des Andersdenkenden; die Integration ist die konkrete Realität derjenigen, die nicht abweichen, nicht anderer Meinung sind. Das Bild der Apokalypse zeichnet sich ab, wenn man die Texte über die Massenkultur liest; das Bild der Integration ersteht bei der Lektüre der Texte aus der Massenkultur.
\end{abstract}

Bezug nehmend auf Eco konstatieren Briese, Faber und Podewski, dass „der Motor apokalyptischen Denkens [...] vor allem von Künstlern und Intellektuellen in Gang gehalten zu werden“33 scheint; folglich wäre Leverkühn prädestiniert für die Rolle des Apokalyptikers, nicht zuletzt weil man durch Zeitbloms Erzählen den Eindruck gewinnt, dass er sich nonkonform verhält und mit der Welt unzufrieden ist. ${ }^{34}$ Der Komponist zeigt in einer seiner wahrscheinlich produktivsten

\footnotetext{
${ }^{29}$ Derrida: Apokalypse, S. 15, Herv. i. O.

${ }^{30}$ Brokoff: Die Apokalypse in der Weimarer Republik, S. 21.

${ }^{31}$ Vgl. ebd., S. 24.

${ }^{32}$ Eco, Umberto: Apokalyptiker und Integrierte. Zur kritischen Kritik der Massenkultur. Übers. v. Max Looser. Frankfurt am Main: Fischer 1984 [Mailand 1964/1978], S. 16, Herv. i. O.

${ }^{33}$ Briese, Faber u. Podewski: Aktualität des Apokalyptischen, S. 17.

${ }^{34}$ Vgl. ebd. Liest man die Ausführungen von Derrida über den apokalyptischen Ton, so scheint nicht nur Adrian Leverkühn für die Rolle des Apokalyptikers prädestiniert zu sein, sondern auch die Musik selbst. Derrida rekurriert mehrfach auf Ausdrücke und Wörter, deren Ursprung der Musik zuzuordnen ist oder auch im musikalischen Bereich verwendet werden und dort nicht selten als Fachbegriffe gelten. Zwar nimmt Derrida auf Kants Ton Bezug, spricht aber gleichzeitig etwa von "différence tonale“, „neutralité du ton“, „,norme atonale“, „tonalité“, „,voix“ und „timbre“, was dem apokalyptischen Diskurs einen
} 
Phasen zwei psychologische Zustände - einen depressiven und einen gehobenen -, die ,innerlich nicht scharf gegen einander abgesetzt waren“ (DF: 512). Während er die Apocalipsis cum figuris komponiert, isoliert er sich von der Welt, ,um unbelauscht, unbeargwöhnt, in ausgeschalteter, von unserem Gesundheitsleben schmerzhaft abgesonderter Verborgenheit Entwürfe zu hegen und zu entwickeln“ (DF: 516). ${ }^{35}$ Adrian Leverkühn komponiert diese „Lebensgeschichte der Musik" (DF: 542) wie besessen, also genau mit jener Besessenheit der dissenters, von denen Eco spricht; dies ist nicht verwunderlich, da er zu diesem Zeitpunkt Esmeralda und dem ,Teufel' bereits begegnet war. Die Apocalipsis wird schnell komponiert: 1919 in einer Zeitspanne von sechs Monaten, ein Jahr nach Ende des Ersten Weltkriegs. Die Krankheit beeinflusst das Komponieren. Man gewinnt den Eindruck, dass Leverkühn persönlich mit dem Teufel, seiner Inspirationsquelle, spricht. ${ }^{36}$ Auch er ist Knecht einer Vision, die er dringend kommunizieren muss, genau wie Johannes in der Offenbarung, der sich als Knecht bezeichnet, der das Wort Gottes über die Zukunft vermittelt. ${ }^{37}$ Mit Johannes teilt Leverkühn auch das „Erhoben-Werden““;8 des Weiteren, darauf verweist Brokoff, ist auch im Fall des Zeugen der biblischen Offenbarung nicht klar, ob er für denjenigen zu halten ist, der die Wahrheit offenbart oder doch für denjenigen, ,dem die Wahrheit offenbart wird“ ${ }^{39}$ Bezüglich Thomas Manns Romans hängt die Antwort auf diese Frage vor allem davon ab, wie man sich der Teufelsproblematik gegenüber positioniert. ${ }^{40}$

Um kurz einige weitere, wiederkehrende inhaltliche Elemente und Figuren von Apokalypsen zu erwähnen, seien hier exemplarisch die babylonische Hure und die Aufforderung des Komms aufgegriffen. „,[D]ie große Erzhure“ (DF: 519) fungiert laut Brokoff als ,zentrale Metapher der Unreinheit“ ${ }^{\star 41}$ und kann der Binäropposition Unreinheit und Reinheit zugeordnet werden, welche „der Unterscheidung von

musikalischen Charakter verleiht. Siehe Derrida, Jacques: D'un ton apocalyptique adopté naguère en philosophie. Paris: Galilée 1983, S. 18-21.

${ }^{35}$ Erwin hebt hervor, dass sich auch Nietzsche in seinen wahrscheinlich produktivsten Phasen isoliert hat. Vgl. Erwin, Andrew: Rethinking Nietzsche in Mann's Doktor Faustus: Crisis, Parody, Primitivism, and the Possibilities of Dionysian Art in a Post-Nietzschean Era. In: Germanic Review 78 (Herbst 2003) H. 4, S. 283-299, hier: S. 284.

${ }^{36}$ Vgl. DF: 523 „Sprich weiter! Sprich nur weiter!“.

${ }^{37}$ Vgl. Brokoff: Die Apokalypse in der Weimarer Republik, S. 18; Offenbarung 1,1.

${ }^{38}$ Ebd.

${ }^{39}$ Ebd., S. 19

${ }^{40}$ Vgl. Kap. 6.

${ }^{41}$ Ebd., S. 16. 
Transzendenz und Immanenz Kontur verleiht““. ${ }^{42}$ Diese fehlt auch in Leverkühns fiktiver Komposition, die sich von Dürers entsprechender Schilderung inspirieren lässt, der sich wiederum auf Ezechiel und auf eine „Portraitstudie einer venezianischen Kurtisane“ (ebd.) stützte, nicht. Zwar wird in Zeitbloms Beschreibung der Komposition Leverkühns nie erwähnt, dass das in der apokalyptischen Verkündung zentrale Verb ,kommen “ im Imperativ verwendet wird, es lässt sich aber gleichwohl eine Parallele zu Derridas Analyse dieses sprachlichen Elements ziehen. Die Worte „Das Ende kommt, es kommt das Ende, es ist erwacht über dich; siehe, es kommt. Es gehet schon auf und bricht daher über dich, du Einwohner des Landes“ (ebd.) besitzen immerhin Aufforderungscharakter, auch wenn die Imperativform nicht in Verbindung mit dem Verb ,kommen " verwendet wird. ${ }^{43}$ Diese Worte werden zunächst vom Erzähler gesungen und dann einem Responsorium abgegeben, das sie „unvergeßlich wiederholt“ (ebd.): Der Aufforderungscharakter ergibt sich daher sowohl aus der Imperativ-Verwendung des im Rahmen einer apokalyptischen Verkündung ebenfalls wichtigen Verbs ,sehen ${ }^{64}$ als auch aus der obsessiven Wiederholung des Zitats durch den Chor sowie aus der vermittelten Botschaft selbst. Zugleich hebt Derrida die Uneindeutigkeit des ,Komm“ - u. a. aus pragmatischen Gründen - hervor, sodass es reduktiv erscheint, diesem apokalyptischen Element nur einen Aufforderungscharakter zuzuschreiben. ${ }^{45}$ Geeignet sei das ,Komm“ jedoch, um ,in allen Tonlagen ausgesprochen“46 zu werden, was sich anhand von Leverkühns Oratorium bestätigen lässt und dort eine gesangliche Realisierung findet. Das ,Komm“ ist Derrida zufolge selbstreferentiell, denn es kündigt „,nicht diese oder jene Apokalypse [...]“ ${ }^{47}$ sondern „,seine differánce selbst“48 an. Derrida führt weiter aus, dass im ,Komm“ „bereits ein gewisser Ton wider[hallt], es ist an sich selbst die Apokalypse der Apokalypse, Komm ist apokalyptisch“. 49

\footnotetext{
${ }^{42}$ Ebd.

${ }^{43}$ Die Vorlage ist auch unterschiedlich: Zeitblom spezifiziert, dass sich hier Leverkühn wie Dürer an Ezechiel und nicht an der Offenbarung orientiert. Siehe DF: 519.

${ }^{44}$ Siehe Brokoff: Die Apokalypse in der Weimarer Republik, S. 19.

${ }^{45}$ Derrida: Apokalypse, S. 83-88.

${ }^{46}$ Ebd., S. 86.

${ }^{47}$ Ebd., S. 88.

${ }^{48}$ Ebd., Herv. i. O.

${ }^{49}$ Ebd., Herv. i. O. Siehe auch Brokoff: Die Apokalypse in der Weimarer Republik, S. 20.
} 


\subsubsection{Die politische Apokalypse: Der Kridwiß-Kreis}

Im zentralen Teil des 34. Kapitels von Doktor Faustus wird der apokalyptische Diskurs, wie in 4.1 bereits angedeutet, politisch dekliniert. Wenn man den Zeitpunkt der histoire betrachtet und diesen mit der entsprechenden historischen Zeit verbindet, ist das alles andere als erstaunlich: „In den apokalyptischen Texten der Weimarer Zeit findet [...] eine Politisierung der Apokalypse statt" ${ }^{50}$ konstatiert Brokoff, der eben diese Texte zum Gegenstand seiner Untersuchung macht. Wie im Fall von Adrian Leverkühns apokalyptischer Komposition lohnt es sich auch bezüglich dieses apokalyptischen Diskurses nicht, sich auf die Suche nach der Transzendenz Gottes zu begeben, denn diese ist ebenfalls „,durch eine andere Transzendenz ersetzt worden“. ${ }^{51}$ Die sogenannte „Fortsetzung“ (DF: 525) des 34. Kapitels präsentiert einige Kernpunkte der Auffassungen eines Kreises von Intellektuellen, die sich in München im Hause des „Graphiker[s], BuchschmuckKünstler[s] und Sammler[s] ostasiatischer Farbenholzschnitte und Keramik“ (ebd.) Sixtus Kridwiß versammeln. Auch hier handelt es sich noch um eine Apokalypse cum figuris: Der Bereich der Kunst ist nicht nur durch den Veranstalter solcher „Round-table-Sitzungen“ (DF: 526), sondern auch durch die Präsenz des „Kunstgelehrte[n] und Dürerforscher[s] Professor Gilgen Holzschuher“ (DF: 528) vertreten, der die Anspielung an Dürer verstärkt und fortsetzt. ${ }^{52}$

Diese „Männer der Bildung, des Unterrichts, der Wissenschaft“ (DF: 530) wehren sich gegen die bürgerliche Tradition und ihrer Werte „,der Bildung, der Aufklärung, der Humanität“ (ebd.) und plädieren für Gewalt, Autorität sowie die Rückkehr zu Prinzipien und politischen Zuständen mittelalterlicher Gesellschaften. Dr. Egon Unruhe setzt sich beispielsweise in seinen Schriften mit der „Rechtfertigung und wissenschaftlichen Verifizierung uralten Sagengutes“ (DF: 527) auseinander und Professor Georg Vogler, der „Literarhistoriker“ (ebd.), mit einer „Geschichte des deutschen Schrifttums unter dem Gesichtspunkt der Stammeszugehörigkeit“" (ebd.). Die Teilnehmer des Kridwiß-Kreises verfolgen sogar das Ziel, „die parlamentarische Diskussion“ (DF: 531) durch „,die Versorgung der

\footnotetext{
${ }^{50}$ Brokoff: Die Apokalypse in der Weimarer Republik, S. 10.

${ }^{51}$ Ebd., S. 11. Zu theologischen Aspekten des Romans siehe: Rohrmoser, Günter: Dekadenz und Apokalypse. Thomas Mann als Diagnostiker des deutschen Bürgertums. Bietigheim/Baden: Gesellschaft für Kulturwissenschaft e. V. 2005; Piccolo, Veronica: L'onnipotenza imperfetta. Teologia secolarizzata nel Doctor Faustus di Thomas Mann. Mailand: Ancora 2010.

${ }^{52}$ Des Weiteren sammelt Kridwiß Holzschnitte, was sich auch mit Dürers Werk verknüpfen lässt.
} 
Massen mit mythischen Fiktionen“ (DF: 532) zu ersetzen und stützen ihre Überlegungen vor allem auf die Réflexions sur la violence von Sorel. ${ }^{53}$ Die immanente Welt deklarieren sie für gescheitert; die zukünftige Welt ist in ihren Vorstellungen „eine alt-neue, eine revolutionär rückschlägige“ (DF: 534); sie hoffen nämlich auf eine ,neuigkeitsvolle[] Rückversetzung der Menschheit in theokratisch mittelalterliche Zustände und Bedingungen“ (DF: 535). Zeitblom kommentiert das so:

Das war so wenig reaktionär, wie man den Weg um eine Kugel, der natürlich herum-, d. h. zurückführt, als rückschrittlich bezeichnen kann. Da hatte man es: Rückschritt und Fortschritt, das Alte und Neue, Vergangenheit und Zukunft wurden eins, und das politische Rechts fiel mehr und mehr mit dem Links zusammen. $(\text { ebd. })^{54}$

In diesem Fall zeichnet sich der apokalyptische Diskurs durch eine Vermischung politischer Stimmen aus. Darüber hinaus berichtet der Erzähler davon, dass sich die Intellektuellen des Kridwiß-Kreises für ein „sacrificium intellectus“(DF: 532), für einen absichtlichen Verzicht auf ihre intellektuellen Fähigkeiten, entschieden hatten. Als Konsequenz wollen sie alles vereinfachen, wie die Gelehrten von Swifts „Grand Academy of Lagado“55 in Gulliver's Travels, die zwecks einer besseren Verständigung Wörter und Rede abschaffen und Dinge auf dem Rücken herumtragen wollen. ${ }^{56}$ Im zentralen Teil des 34 . Kapitels profiliert sich eine politische Revolution besonderer Art, die in Hinblick auf die programmatisch

\footnotetext{
${ }^{53}$ Vgl. Borchmeyer, Dieter: Mythos. In: Borchmeyer, Dieter u. Victor Žmegać (Hrsg.): Moderne Literatur in Grundbegriffen. Berlin: de Gruyter 1994 [1987], 2. neu bearb. Aufl., S. 292-308, hier: S. 298 f.

${ }^{54}$ Die Metapher des Wegs um die Kugel kommt erneut im dritten Teil des Kapitels vor, in dem Serenus Zeitblom behauptet, dieser sei ,durch ein neuigkeitsvolles Zurückgehen über Bachs und Händels bereits harmonische Kunst hinaus in die tiefere Vergangenheit echter Mehrstimmigkeit“ (DF: 540) verwirklicht. Die Musik Leverkühns strebe nicht nach den ,harmonischen“ Errungenschaften der Musik von Bach und Händel, sondern lasse sich von einer mehrstimmigen Vergangenheit inspirieren. Auch die Gelehrten des Kreises verzichten auf die errungene Zivilisation, um nach einer mythischen und gewaltsamen Vergangenheit zu streben. Siehe dazu Puschmann, Rosemarie: Magisches Quadrat und Melancholie in Thomas Manns Doktor Faustus. Von der musikalischen Struktur zum semantischen Beziehungsnetz. Bielefeld: AMPAL 1983, S. 104.

${ }^{55}$ Swift, Jonathan: Gulliver's Travels. In: Ders.: The complete works of Jonathan Swift, D.D., and Dean of St. Patrick's, Dublin. Containing interesting and valuable papers not hitherto published. And an autograph letter. With memoir of the author. Hrsg. v. Thomas Rescoe, Bd. 1. London: Bell and Daldy 1869 [1726], S. 1-81, hier: S. 48.

${ }^{56}$ Vgl. ebd.: S. 50; DF: 536.
} 
deklarierte Absicht einer Rückkehr zu den Ursprüngen als ,,re-volution“57 aufgefasst werden könnte und ,präfaschistisch“淿 ${ }^{\text {anmutet. }}{ }^{59}$ Das Ende der Immanenz, also die Vernichtung der Differenz ist auch wie in Leverkühns Oratorium und in vielen apokalyptischen Texten wesentlicher Bestandteil dieses apokalyptischen Diskurses, der eine politische und gesellschaftliche Transzendenz vorsieht, die aus Gewalt, Mythen, Diktatur und Differenzlosigkeit besteht. Gewalt ist apokalyptischen Schriften nicht fremd: Brokoff weist darauf hin, dass in der Offenbarung des Johannes „Wahrheit und Gewalt selbst auf unlösliche Weise miteinander verknüpft“60 sind, da „Gott und Gewalt ineinsgesetzt“61 werden. Nicht nur in den Konversationen des Kridwiß-Kreises kristallisiert sich also Gewalt als notwendige Voraussetzung zur Erreichung der Transzendenz heraus, sondern auch in dem biblischen, apokalyptischen Text par excellence.

Als Apokalyptiker lassen sich auch wie der Zeuge von Leverkühns Komposition die Teilnehmer des Kridwiß-Kreises bezeichnen, denn sie beabsichtigen nicht, sich zu integrieren, sondern bilden Theorien über den Zerfall der Gesellschaft aus. Ebenfalls könnte man sie mit den Mystagogen nach Derrida in Verbindung bringen, die zum Delirium, ${ }^{62}$ zu jeder ,Verstimmung der Saiten und Stimmen im Kopf "63 führen, da sie die Werte, die Grundprinzipien und die politischen Institutionen einer bestehenden Gesellschaft in Frage stellen. Aus Sicht der Intellektuellen des Kridwiß-Kreises stellt die Vernichtung der Differenz als Voraussetzung einer geschichtlichen Erlösung etwas Positives dar: Ihre Ideen können dem Typ einer „Erfüllungs- und Erlösungsapokalypse“64 zugeordnet werden und historisch mit jenen politischen Bewegungen u. a. in Deutschland assoziiert werden, die den ideologischen Boden für den Nationalsozialismus bereiten.

Auch dieser politisch deklinierte apokalyptische Diskurs, der sich im Rahmen der Sitzungen des Kridwiß-Kreises entfaltet und von dem Zeitblom als -

\footnotetext{
${ }^{57}$ Roberts, David: The Sense of an Ending. Apocalyptic Perspectives in the 20th-century German Novel. In: Orbis Litterarum 32 (1977) H. 2, S. 140-158, hier: S. 147.

${ }^{58}$ Petersen, Jürgen H.: Der unzuverlässige Narrator. Figuren-Erzählen in Thomas Manns Doktor Faustus. In: Revista de Filología Alemana 16 (2008), S. 165-187, hier: S. 177.

${ }^{59}$ Nicht unangebracht scheint eine Verbindung der Ideen des fiktiven Kridwiß-Kreises zu denen der konservativen Revolution. Vgl. Mohler, Armin: Die konservative Revolution in Deutschland 1918-1932. Grundriß ihrer Weltanschauungen. Stuttgart: Vorwerk 1950.

${ }^{60}$ Brokoff: Die Apokalypse in der Weimarer Republik, S. 24.

${ }^{61}$ Ebd., S. 22.

${ }^{62}$ In diese Deutungsperspektive lässt sich Leverkühns Delirium gut einordnen. Vgl. Kap. 6.

${ }^{63}$ Derrida: Apokalypse, S. 34, vgl. auch S. 27-34.

${ }^{64}$ Briese, Faber u. Podewski: Aktualität des Apokalyptischen, S. 21.
} 
nach seinen Worten - passiver Beobachter berichtet, ${ }^{65}$ ist mit dem Ende des 34. Kapitels keineswegs beendet. Die Teilnehmer des Kreises spekulieren über die Möglichkeiten eines Auswegs aus einem Krisenzustand, der die Politik, die Gesellschaft und, nicht zuletzt, auch die Wissenschaft betrifft. Dieses Klima spiegelt sich laut Zeitblom in Leverkühns Oratorium wider, das ,mit dem bei Kridwiß Gehörten in eigentümlicher Korrespondenz, im Verhältnis geistiger Entsprechung stand“ (DF: 539). Die ideologischen Auffassungen des Kridwiß-Kreises verdeutlichen ein ,prinzipielle[s] Nicht-Einverständnis“66 mit den Werten und Prinzipien ihrer Zeit. Im Gegensatz dazu entspringt Leverkühns Komposition ,einer Rebellions- und Protesthaltung “67 eben gegen jene ideologischen Positionen, die sich damals verbreiteten, und wählt als Rebellions- und Warnungswaffe das Medium der Musik. Das 34. Kapitel enthält eine Verschachtelung apokalyptischer Diskurse; im Fall der Komposition Leverkühns ist die Apokalypse auch histoire des musikalischen Werkes selbst.

Börnchen stellt in seiner Dissertation fest, dass ,,sich in den letzten Jahrzehnten der Tenor durchgesetzt zu haben“68 scheint, ,den Roman als ,Apokalypse ‘ zu lesen - eine Interpretation, zu der Humor und Komik nicht zu passen scheinen“. 69 In dieser Hinsicht könnte man der vorliegenden Studie ebenfalls vorwerfen, sich in diese Interpretationslinie einzuschreiben und dementsprechend den Humor und die Komik von Doktor Faustus zu übersehen. An dieser Stelle muss aber beachtet werden, dass Apokalypse mit Weltuntergang nicht gleichzusetzen ist, obwohl sie in der Alltagskommunikation, in literarischen Texten, in Filmen und in der Kunst oft so verstanden wird. ${ }^{70}$ Wie zu Beginn des vorliegenden Abschnitts bereits erwähnt, unterstreicht Brokoff gleich am Anfang seiner Studie, dass auch in der Offenbarung des Johannes der Weltuntergang ,keineswegs den Schlußpunkt" ${ }^{\text {71 }}$ der Erzählung bildet. Des Weiteren weisen Briese, Faber und Podewski darauf hin, dass der Begriff ,Katastrophe‘, der mit dem ,Weltuntergang ' in apokalyptischen Texten eng verquickt ist und den heute kaum jemand mit etwas

\footnotetext{
${ }^{65}$ Vgl. DF: 534.

${ }^{66}$ Briese, Faber u. Podewski: Aktualität des Apokalyptischen, S. 9.

${ }^{67}$ Ebd.

${ }^{68}$ Börnchen: Kryptenhall, S. 92 f.

${ }^{69}$ Ebd. Ein Beispiel für diese Deutungslinie ist etwa der Aufsatz Mehrings: Mehring, Reinhard: Apokalypse der deutschen „Seele“? Thomas Manns „Doktor Faustus“ als „Zeitroman“. In: Weimarer Beiträge 51 (2005) H. 2, S. 188-205.

${ }^{70}$ Brokoff: Die Apokalypse in der Weimarer Republik, S. 7.

${ }^{71}$ Ebd.
} 
Positivem assoziieren würde, ursprünglich im Sinne einer „Wendung zu Besserem“"72 eine positive Bedeutung trug, die „,von der römischen Antike bis über die Renaissance hinaus" ${ }^{\text {73 }}$ auch so verwendet wurde. Dies gelte auch für den Begriff ,Apokalypse“, der - „bis in die Frühe Neuzeit hinein - immer auch ein positiv besetzter Erwartungs- und Hoffnungsbegriff ${ }^{* 74}$ gewesen sei. Dies änderte sich erst „,mit den allmählichen Transformationen des Christentums“. ${ }^{75}$ Die drei Forscher*innen bestätigen die von Brokoff ebenfalls vorgestellte „,binäre Bedeutungsstruktur“76 der Apokalypse, die aus „Krise, Untergang und Erlösung“77 besteht. Da Leverkühns Apocalipsis cum figuris vor allem mittelalterliche und frühneuzeitliche Texte und Kunstwerke als Vorlage aus der Absicht heraus verwendet, Altes und Neues musikalisch zu kombinieren, scheint sein Werk sowohl in der musikalischen Faktur als auch in seiner Darstellung der Apokalypse die ursprüngliche Bedeutung des Begriffs zu beleben: Den verwendeten Texten ist diese Bedeutung inhärent. Eine Apokalypse wie Leverkühn sie nach Zeitbloms Wiedergabe versteht, schließt keineswegs Positives aus, sondern impliziert sogar eine Erlösung.

Von der Parodie verschiedener Musikstile war in diesem Kapitel bereits die Rede. Dazu sei ergänzt, dass Zeitblom im 34. Kapitel die provozierende Haltung von Künstler*innen adressiert. Nachdem der Erzähler über den Namen „OberKirchenrat" (DF: 541) berichtet, den Richard Wagner zur Zeit des Parsifal unter einem Brief hinzufügte, ist im Text folgender Kommentar des Erzählers zu finden:

Für den Nicht-Künstler ist es eine recht intriguierende Frage, wie ernst es dem Künstler mit dem ist, was ihm das Angelegentlich-Ernsteste sein sollte und zu sein scheint; wie ernst er sich selbst dabei nimmt und wieviel Verspieltheit, Mumschanz [sic], höherer Jux dabei im Spiele ist. (DF: 541$)^{78}$

Ironie und Humor sind aus der Komposition Leverkühns nicht wegzudenken, auch wenn ihre Basis das Apokalyptische ist. Die im 34. Kapitel dargestellten Ereignisse stammen zudem aus Zeitbloms fiktiver Feder, dem „als Nachfahre der deutschen Humanisten“ (DF: 12) die ursprüngliche Bedeutung des Wortes ,Apokalypse ' bestimmt nicht fremd ist. Da er erzählt, ist er derjenige, der

\footnotetext{
${ }^{72}$ Briese, Faber u. Podewski: Aktualität des Apokalyptischen, S. 31.

${ }^{73}$ Ebd.

${ }^{74}$ Ebd.

${ }^{75}$ Ebd.

${ }^{76}$ Ebd.

${ }^{77}$ Ebd.

${ }^{78}$ Dazu siehe auch Rohrmoser: Dekadenz und Apokalypse, S. $214 \mathrm{f}$.
} 
den apokalyptischen Ton setzt: Es scheint legitim zu fragen, ob er der Apokalyptiker par excellence des Romans sei. Mit der Verstimmung, die Derrida zufolge apokalyptischen Diskursen zugrunde liegt, scheint sich der Viola d'amoreSpieler ebenfalls gut auszukennen. ${ }^{79}$ Zeitblom rezipiert Leverkühns Werk: Briese, Faber und Podewski heben hervor, dass ,,nicht die Produzenten Apokalypsen produzieren, sondern die Rezipienten“. 80 Dies verweist auf Derridas Auffassung, wonach es eine wichtige aber nicht immer selbstverständliche Voraussetzung für das Gelingen der apokalyptischen Kommunikation ist, dass der Ton angenommen wird: ,Wer den apokalyptischen Ton annimmt, wird Ihnen [sic] etwas bedeuten, wenn nicht gar sagen“. ${ }^{11}$ Der Erzähler rezipiert Leverkühns Oratorium allerdings nicht passiv, sondern produziert seinerseits seine apokalyptische, verstimmte Darstellung des Lebens des Freundes. Dass „die Apokalypse von langer Dauer"82 ist, bestätigt das 34. Kapitel von Thomas Manns Doktor Faustus durch das komplexe Ineinandergreifen ${ }^{83}$ zahlreicher apokalyptischer Diskurse auf der intradiegetischen und extradiegetischen Ebene der Narration und darüber hinaus. ${ }^{84}$

\footnotetext{
${ }^{79}$ Vgl. Derrida: Apokalypse, S. 62 f. Auch in der Originalausgabe erscheint das Wort auf Deutsch: Derrida spricht von einer „Verstimmung multipliant les voix et faisant sauter les tons, ouvrant chaque parole à la hantise de l'autre dans une polytonalité immaîtrisable, avec greffes, intrusions, parasitages“. Derrida: D'un ton apocalyptique, S. 67, Herv. i. O. Bemerkenswert ist hier, dass wieder Begriffe verwendet werden, die auch im musikalischen Bereich Verwendung finden. Siehe Fußnote 34. Hier wird das Thema kurz angesprochen, ausführlicher wird es im siebten Kapitel der vorliegenden Arbeit behandelt.

${ }^{80}$ Briese, Faber u. Podewski: Aktualität des Apokalyptischen, S. 15.

${ }^{81}$ Derrida: Apokalypse, S. 64.

${ }^{82}$ Ebd., S. 75.

${ }^{83}$ Wohl könnte man auch sagen, dass diverse apokalyptische Diskurse im Roman montiert werden, und sich damit auf eine Technik berufen, nämlich die Montage-Technik, die in der Doktor Faustus-Forschung zum Untersuchungsgegenstand bereits gemacht wurde. Siehe etwa Kropfinger, Klaus: „Montage“ und „Composition“ im „Faustus“ - Literarische Zwölftontechnik oder Leitmotivik? In: Röcke, Werner (Hrsg.): Thomas Manns Doktor Faustus, S. 345-368.

${ }^{84}$ Brokoff kritisiert Derridas Auffassung einer Zwangsläufigkeit der Fortsetzung des apokalyptischen Diskurses, denn die Ebene der Transzendenz setze dem apokalyptischen Diskurs ein Ende. Brokoff unterstreicht somit ,die endliche Struktur der apokalyptischen Entscheidung“, die „das unendliche Sprechen gewaltsam beendet“. Angesichts der Überfülle an Werken, die sich mit dem apokalyptischen Diskurs auseinandersetzen, und der immer noch bestehenden Faszination für die Apokalypse könnte man wagen, Derridas und Brokoffs Thesen miteinander zu kombinieren und folglich für die Mittelposition eines vorläufigen Endes argumentieren. Zwar beendet die apokalyptische Entscheidung das Sprechen, das
} 
Anhand des 34. Kapitels von Doktor Faustus kann den vorigen Ausführungen entsprechend der apokalyptische Diskurs des Romans in seinen musikalisch- und politisch-bezogenen Deklinationen näher beleuchtet werden. Auf einer Makroebene der Analyse geschieht das durch die Definition der Art von Apokalypse: Als Voraussetzung gilt die Identifikation der Stadien der Immanenz und der Transzendenz sowie die Untersuchung der verwendeten Quellen. Auf einer Mikroebene ist diese Untersuchung durch die Analyse der Präsenz und Realisierung von wiederkehrenden Elementen apokalyptischer Reden, z. B. dem ,Komm'-Motiv und der Figur des Zeugen, möglich. Dieses analytische Prozedere wird auch auf die darauf folgenden Kompositionen von Boehmer, Kurz und Searle angewendet und mit Kategorien aus der Intermedialitätsforschung stärker in Verbindung gebracht.

\subsection{Vom Roman zur Musik}

Der vorliegende Abschnitt integriert die literaturzentrierte, intermediale Analyse des ersten Abschnitts in die Analyse der intermedialen Transpositionen bzw. Bezugnahmen jeweils von Konrad Boehmer (Apocalipsis cum figuris), KarlWieland Kurz (Apocalipsis sine figuris) und Humphrey Searle (Apocalypsis cum figuris). Somit sollen einerseits der Transfer und die Transformation von Leverkühns Oratorium vom Medium der fiktionalen Schrift in das Medium der Musik, andererseits der apokalyptische Diskurs der jeweils betrachteten Komposition rekonstruiert werden. $\mathrm{Zu}$ diesem letzten Zweck wird erneut auf die Terminologie und die Positionen des vorigen Abschnitts rekurriert.

\subsubsection{Inverse Warnapokalypsen: Konrad Boehmers Apocalipsis cum figuris}

Die erste Komposition, die hier präsentiert wird, ist die Apocalipsis cum figuris von Konrad Boehmer (1941-2014) für vier Schlagzeuge, zwei Klaviere, drei Pop-Vokalist*innen und vier Lautsprecher, die 1984 anlässlich der Donaueschinger Musiktage aufgeführt wurde. ${ }^{85}$ Während der niederländische Komponist, der

stellt aber ein vorläufiges Stadium dar. Brokoff: Die Apokalypse in der Weimarer Republik, S. 26 (siehe auch S. 25).

${ }^{85}$ Die Partitur wurde 1984 vom Tonos Musikverlag (Darmstadt) veröffentlicht, kann aber auch aus der Webseite der Konrad Boehmer Foundation heruntergeladen werden. Siehe Boehmer, Konrad: Apocalipsis cum figuris (Partitur). In: „Konrad Boehmer Foundation“. $<$ https://www.kboehmer.nl/wp-content/uploads/2019/12/Apocalipsis.pdf > (letzter 
in Berlin geboren wurde und in Köln mit einer Dissertation zur Theorie der offenen Form in der Neuen Musik im Fach Musikwissenschaft promovierte, ${ }^{86}$ an einer Oper über den Faust-Stoff, die sich aber nicht mit Manns Roman auseinandersetzte, arbeitete, wurde er zu einem Symposium über Thomas Manns Doktor Faustus nach Brüssel eingeladen. Diese Einladung war für Boehmer der Anlass, den Roman nach vielen Jahren erneut zu lesen. Das in 4.1.1 erwähnte Zitat über die umgekehrte Rolle und Funktion der Dissonanz und des Tonalen und folglich den paradoxen Charakter von Leverkühns Oratorium, ${ }^{87}$ das eben das Tonale und nicht die Dissonanz mit der Hölle assoziiert, versteht der Komponist als die Essenz des Werkes. ${ }^{88}$

Bei der Lektüre dieser wohl faszinierendsten Beschreibung [...] fesselte mich vor allem die Radikalität des Entwurfs, die ein Menschenalter nach dessen Niederschrift - und grade [sic] heute, in der Ära musikalischen Neo-Biedermeiers - von ungebrochener Aktualität ist.

Diese Aussage verdeutlicht nochmals, was im vorigen Abschnitt bereits hervorgehoben wurde: Leverkühns fiktive Komposition enthält musikalische Ideen und Ansätze, die auffällige Korrespondenzen zu musikalischen Ideen, Ansätzen und Versuchen nicht nur seiner Zeit, sondern auch der Nachkriegszeit aufweisen.

Konrad Boehmer erläutert im Programm zur Uraufführung, seine Intention sei nicht gewesen, Leverkühns Oratorium nachzukomponieren, um eine Art Programm-Musik zu konzipieren. Die ästhetische Konzeption und die Spannweite der im Roman geschilderten Komposition seien jedoch durchaus die Grundlagen seines Werkes gewesen. Wichtiger Ausgangspunkt seiner Überlegungen sei auch die Faszination für den Konnex zwischen Zivilisationsstadien der europäischen Geschichte und „apokalyptischen Ideologien“. ${ }^{89}$ Direkt oder indirekt scheint sich hier der Komponist auf die vorher erwähnten Ausführungen von Umberto Eco zur Wirkung der Argumentationen von Apokalyptiker*innen zu berufen, in denen die Massenkultur als Antikultur erscheint: Diese Art des Argumentierens ziele auf eine Reaktion der Integrierten und folglich der Massenkultur, die somit von den

Zugriff: 21.08.2020). Eine Aufnahme des Stückes findet man in der CD Acousmatrix 5 - history of electronic music V, Amsterdam: BVHaast (Nr. 9011) 1990.

${ }^{86}$ Siehe Boehmer: Zur Theorie der offenen Form in der Neuen Musik. Darmstadt: Tonos 1967.

${ }^{87}$ Vgl. DF: 544.

${ }^{88}$ Boehmer: Apocalipsis cum figuris. In: Programmheft der Donaueschinger Musiktage 1984, S. 23-26, hier: S. 23.

${ }^{89}$ Ebd., S. 24. 
Apokalyptiker*innen beeinflusst werden, ab. Dies ist laut Eco äußerst produktiv, denn es setzt Prozesse in Gang, die zu einer Erweiterung der Kultursphäre und einer „Zirkulation einer ,populären“ Kunst und Kultur“90 beitragen. Die Prozesshaftigkeit, die Eco somit anspricht und zu den konstitutiven Merkmalen der apokalyptischen Verkündung als Akt des Offenbarens zählt, spielt eine vordergründige Rolle in Boehmers Komponieren. Der Komponist sieht eigenen Aussagen zufolge in all seinen elektronischen Werken ,das Moment des Klanges als Proze $\beta$ (und nicht: als Objekt) "91 an: Das Ergebnis der Klangerzeugung ist für ihn nachrangig, während ihre Prozesshaftigkeit eine Sonderstellung einnimmt. Außerdem beschäftigt sich Boehmer - genau wie der fiktive Komponist Adrian Leverkühn im apokalyptischen Oratorium- mit dem Problem der Denaturierung des Klanges und ist der Auffassung, dass gerade in Manns Doktor Faustus schon eine Lösung gefunden werden könne: ${ }^{92}$

Die Lösung der ästhetischen Aporien, die, wie wir jetzt wissen, nicht darin bestehen konnte, daß man willkürlich jeden „,natürlichen“ Klang in den Fleischwolf der Klänge, den Ringmodulator, stopfen konnte, jene Lösung hätte man damals schon bei Thomas Manns Leverkühn nachlesen können, der den „,denaturierten“ Klang als musikgeschichtliches Phänomen schlechthin begriff [...].

Deshalb sei Leverkühn ,der letzte und radikalste Repräsentant westlicher KunstMusik“,93 da er „,den Kunst-Laut in den Natur-Laut zurück zu versenken“94 versucht und damit „etablierte[] Systeme und ästhetische[] Normen“95 sprengt. Der Komponist assoziiert eben aufgrund dieser Überwindung von inneren Grenzen der Musik das fiktive Projekt Leverkühns mit dem Espace von Edgar Varèse, der die äußeren Grenzen der Musik (des Konzertsaals) sprengt. Dadurch schlägt er ein weiteres Modell für Leverkühns fiktive Komposition vor, das allerdings in Thomas Manns Tagebüchern und Selbstkommentaren nicht erwähnt wird und sich angesichts der Entstehungszeit (ca. 1947) eher mit der Entstehungszeit des Romans als mit der von Leverkühns Apocalipsis in Verbindung bringen lässt. ${ }^{96}$

\footnotetext{
${ }^{90}$ Eco: Apokalyptiker und Integrierte, S. 16; vgl. dazu auch S. 15.

${ }^{91}$ Boehmer: Apocalipsis (Programmheft), S. 23.

${ }^{92}$ Ebd.; siehe DF: 542.

${ }^{93}$ Boehmer: Apocalipsis (Programmheft), S. 24.

${ }^{94}$ Ebd.

${ }^{95}$ Ebd.

${ }^{96}$ Siehe Griffiths, Paul: Varèse, Edgar [Edgar] (Victor Achille Charles). In: Grove Music Online. Zuerst veröffentlicht 20.01.2001, online veröffentlicht 2001.< https://doi.org/10. 1093/gmo/9781561592630.article.29042 > (letzter Zugriff: 21.08.2020).
} 
Die Unmöglichkeit einer Zuordnung von Leverkühns Kompositionstendenzen in der Apocalipsis zu denen des musikalischen Panoramas seiner Zeit wäre nicht nur ein Beweis für die Fiktionalität des Textes, sondern könnte auch eine Erklärung dafür liefern, warum manchmal bei Komponist*innen der Eindruck entsteht, dass in Doktor Faustus Tendenzen der Neuen Musik der Nachkriegszeit antizipiert werden. ${ }^{97}$ Leverkühns Oratorium weist laut Boehmer auf das Ende einer musikalischen Ära - der bürgerlichen Epoche - hin und ,dieses Ende nimmt bei ihm apokalyptische Dimensionen an, wird Ausdruck des freien Leidens, ,musique concrète" im wahrsten Sinne des Wortes". ${ }^{98}$ Auch der Komponist scheint daher in diesem Zitat Leverkühns Oratorium dem apokalyptischen Stadium des Untergangs einer musikalischen Welt zuzuordnen, weil es gleichzeitig durch etwa die Denaturierung des Klanges auf eine neue Welt hinweise, aber im Ganzen immer noch nicht dem eingetretenen Stadium der musikalischen Transzendenz entspreche.

Die Präsenz der Pop-Sänger*innen sollte für diejenigen, die mit dem Denken Boehmers vertraut sind, keine Überraschung sein. In seinem Essay Reihe oder Pop? erläutert der Komponist, er habe großen Respekt vor Bob Dylan, Ferré, den Lords und den Rolling Stones, weil sie durch ihre Musik Vorurteile thematisiert und entkräftet haben, und offenbart den programmatischen Versuch, eine Annäherung der Neuen Musik an die Pop-Musik zu fördern: ${ }^{99}$

Man könnte den Versuch wagen, eine Synthese zwischen den sympathischen Songs und dem musikalischen Bewußtsein zu bilden, das wir unbeirrt für das authentischere und humanere halten.

Diese Idee einer Kombination verschiedener Musikstile und Musikgattungen überschneidet sich mit der musikalischen Konzeption von Leverkühns Apocalipsis: Es handelt sich in diesem Fall um den Transfer eines in der literarischen

\footnotetext{
${ }^{97}$ Dazu vgl. auch Manzonis Position (5.2.1.1).

${ }^{98}$ Boehmer: Apocalipsis (Programmheft), ebd.

${ }^{99}$ Ders.: Reihe oder Pop? (1966) In: Ders.: Das böse Ohr. Hrsg. v. Burkhardt Söll. Köln: Du Mont 1993, S. 96-116, hier: S. 113. Erwähnenswert ist in diesem Kontext auch die biographische Information, dass sich Konrad Boehmer in den 1960er und 1970er Jahren gegen Stockhausen, Kagel und andere Komponisten der Zeit positionierte. Vgl. Sabbe, Herman: Boehmer, Konrad. In: Grove Music Online. Zuerst veröffentlicht 20.01.2001, online veröffentlicht 2001.<https://doi.org/10.1093/gmo/9781561592630.art icle.03375 > (letzter Zugriff: 21.08.2020).
} 
Vorlage durch die Technik des intermedialen telling beschriebenen musikkompositorischen Verfahrens in das Medium der Musik, also um die Medientransformation einer intermedialen Bezugnahme des Textes, die selber einer Medientransformation entspringt. Man könnte in diesem Fall von einer Intermedialität zweiten Grades oder einfach von einer Rückkehr zum ursprünglichen Medium sprechen: Der zweite Schritt jedoch der Bezugnahme in Doktor Faustus beeinflusst immerhin diese Rückkehr. Nicht nur das fiktive, konstitutive Verfahren von Leverkühns Komposition wird bei Boehmer in die Musik transferiert, es werden auch die musikalischen Vorbilder übernommen, da sich sowohl Leverkühn als auch Boehmer auf populäre Musikgattungen stützen. Zugleich lassen sich beide Werke aufgrund jener der kompositorischen Konzeption zugrunde liegenden „Vermischung der Stimmen, Gattungen und Codes"100 weit über paratextuelle Hinweise hinaus dem apokalyptischen Diskurs zuordnen: Briese, Faber und Podewski weisen darauf hin, dass Paratexte besonders im Fall von apokalyptischen Texten oft eines Vergleichs mit der inhaltlichen Ebene bedürfen. ${ }^{101}$

Boehmers Komposition gliedert sich in vier Ebenen, welche die figurae der Komposition darstellen. Die erste Ebene bildet „das ,Geheul schreiender Menschen' im Griff der apokalyptischen Würgengel“102 ab und wird musikalisch mithilfe von „körperlichen“, konkreten Klängen realisiert. Die zweite präsentiert apokalyptische Bilder und Visionen, ohne eine Geschichte erzählen zu wollen: Vielmehr erwähnt sie Textfragmente und wiederholt sie. Es sind selbstverständlich Teile der Offenbarung des Johannes enthalten, in Boehmers „Resumé aller Verkündigungen des Endes“ (DF: 520) kommt aber auch eine Vielzahl anderer Texte und Autor*innen vor, die man in Doktor Faustus nicht findet, wie z. B. Barockgedichte über das Vanitas-Motiv, Hölderlin, der die Parzen bittet, ihm noch einen letzten Sommer zu gewähren, Giacomo Leopardi, dessen Aussage „Die Welt ist Dreck" eine zentrale Rolle spielt, weil sie das Stück beschließt, und ein Text von Karl Marx, der den Kapitalismus kritisiert. Die babylonische Hure wird musikalisch mithilfe von Szenen aus dem Roman Juliette ou les prospérités du vice des Marquis de Sade porträtiert. Die Vorlage ist also nicht mehr bzw. nicht nur die babylonische Hure von Dürer, der sich seinerseits am Porträt einer venezianischen Kurtisane orientierte. ${ }^{103}$

Die dritte Ebene ordnet Boehmer ironischerweise der geordneten, zivilisierten Welt zu, die durch die Einführung von Nationalhymnen ins Lächerliche

\footnotetext{
${ }^{100}$ Derrida: Apokalypse, S. 76.

${ }^{101} \mathrm{Vgl}$. 4.1.

${ }^{102}$ Boehmer: Apocalipsis (Programmheft), S. 24.

${ }^{103}$ Vgl. DF: 519.
} 
gezogen wird. ${ }^{104}$ Diese Ebene der Komposition kann mit der Fortsetzung des 34. Kapitels von Thomas Manns Doktor Faustus in Verbindung gebracht werden, da die Nationalhymnen auf den Nationalismus anspielen: Aufgrund des Erscheinungsjahres schließt Boehmers Komposition den Bezug auf zeitgenössische Nationalismen mit ein. Die vierte Ebene wird dem Teufel gewidmet; wie in Dantes Inferno begegnet man ihm im letzten girone. Die Rolle des Teufels übernehmen die Pop-Vokalist*innen. Sie sollten die ,schändlichen Liederreigen der Söhne des Pfuhls“ (DF: 545) darstellen. Gegen Ende des Stückes findet sich eine Mischung aus Choral und Hitsong, die um das tonale Zentrum von C-Dur kreist. ${ }^{105}$ Diese ,einfache“ Tonalität - ein möglicher Bezug auf die Realisierung der Hölle durch das Tonale bzw. das Banale in der Komposition Leverkühns begleitet den Text „I am a dream - I am God“, 106 eine Aussage des russischen Komponisten Skrjabin, die mit dem Delirium assoziiert werden könnte, das apokalyptische Verkündungen bewirken und dem Adrian Leverkühn selbst zum Opfer gefallen ist. ${ }^{107}$ Die Aufnahme des ersten Schreis eines neugeborenen Kindes, den man ganz am Ende der Komposition zu hören bekommt, wird mit den folgenden Worten Leopardis verbunden: „Nur Bitterkeit und Langeweile - Die Welt ist Dreck“ (S. 56). Ordnet man aber diese letzten Elemente der Komposition in den apokalyptischen Diskurs ein, so ist nicht nur die scheinbar einfache CDur-Tonart alles andere als erstaunlich. Boehmers Komposition schafft in einem einzigen Stück, was dem fiktiven Leverkühn erst mit der Weheklag gelingt, nämlich den Sprung zur Transzendenz. Die Differenz wird vorläufig vernichtet und eine einfache Tonart herrscht; ${ }^{108}$ darüber hinaus ist Skrjabins Aussage, „I am a dream - I am God“, ein sprachlicher Verweis auf eine Traum-Dimension und auf Gott selbst. Leopardis Worte erklären die immanente Welt für endgültig gescheitert und der erste Schrei des neugeborenen Kindes übernimmt die Funktion jenes „hohe[n] g eines Cello“ (DF: 711), das den Neubeginn markiert. Alles erreicht morendo ein Ende, was dem apokalyptischen Diskurs ein vorläufiges Ende setzt (Abbildung 4.1).

\footnotetext{
${ }^{104}$ Ebd., S. 25.

${ }^{105}$ Boehmer: Apocalipsis (Partitur), S. 50-53.

${ }^{106}$ Ebd., S. 53.

${ }^{107}$ Vgl. Derrida: Apokalypse, S. 33 u. Kap. 6.

${ }^{108}$ Das betrifft nur S. 50-53 der Komposition und nicht das Notenbeispiel dieses Unterabschnitts; bemerkenswert ist auf S. 50-53, dass im Gegensatz zu Leverkühns Weheklag das tonale System wieder eingeführt wird, was beweist, dass Boehmers Komposition als sekundäres intermediales Phänomen aus anderen musikgeschichtlichen Prämissen entstand.
} 


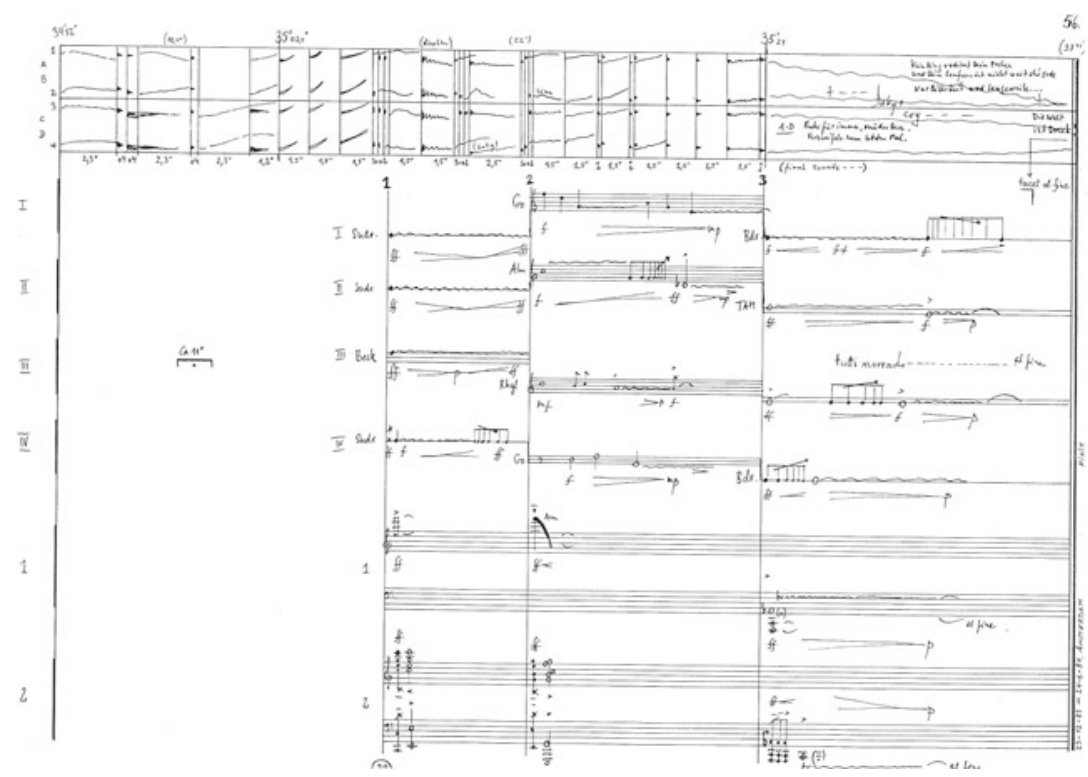

Abbildung 4.1 Das Ende des Stückes (S. 56). Mit freundlicher Genehmigung der Konrad Boehmer Foundation

Dekonstruktivistische Verfahren, besonders die „Dekonstruktion des Gegensatzes"109 lassen sich auch hier wie im vorigen Kapitel in Odegards The Calling of St. Gregory beobachten. Der Pessimismus, welcher Leopardis Zitat zum Ausdruck bringt, scheint durch den Schrei des neugeborenen Kindes in Hoffnung umgewertet zu werden. ${ }^{110}$ Gegensätze werden sowohl in Leverkühns Apocalipsis als auch in Boehmers gleichnamiger Komposition dekonstruiert. Der Autor erklärt wie folgt seine Poetik: ${ }^{111}$

Das Schlechte ist mir gut (ich umarme den Dreck), das Gute ist mir schlecht (ich verachte Moralisten); Ordnung ist mir Chaos (denn leerer als absolute Ordnung kann selbst das Chaos nicht sein); Chaos zu schaffen, ist in meinen Kompositionen

\footnotetext{
${ }^{109}$ Derrida: Positionen, S. 66.
}

${ }^{110}$ Allerdings könnte es auch sein, dass der hoffnungsvolle Schrei des Kindes in Pessimismus umgewertet wird, was bedeuten würde, dass der apokalyptische Diskurs der Komposition in eine pessimistische Transzendenz mündet.

${ }^{111}$ Ebd., S. 25. 
Anlaß zu strengster musikalischer Ordnung; Untergang ist mir Erneuerung (ich bin fortschrittsgläubig), Erneuerung Untergang (da bin ich Romantiker).

Nach der Analyse von Boehmers Apocalipsis cum figuris wird im Folgenden auf die Rezeption des Werkes durch die Besprechung einiger Rezensionen und wissenschaftlicher Artikel kurz eingegangen. Der Kritiker Ernst Vermeulen, der eine Aufführung des Stückes im Amsterdams Concertgebouw hörte, sagte, die Komposition sei so dämonisch wie wenige andere in der Musikgeschichte. ${ }^{112}$ Nach einer sehr kurzen Analyse der Komposition konstatiert der Literaturwissenschaftler Theodore Ziolkowski, dass Boehmer den Beschreibungen in Thomas Manns Roman nicht präzise folge, aber das Gefühl von Barbarei effektiv vermittle. ${ }^{113}$ Der Musikwissenschaftler und Komponist Alfred Zimmerlin beschreibt die Apocalipsis cum figuris als das extremste Werk Boehmers, ,ein fast vierzigminütiges Pändamonium von einer Radikalität, die gewiss über das hinausgeht, was Thomas Mann seinem Adrian Leverkühn im Doktor Faustus andichtete".114 Offenbar treten durch diese Rezensionen Wertungen zutage. Zudem werden voreilige Schlussfolgerungen über komplexe Dynamiken und Implikationen von intermedialen Transpositionen gezogen, die u. a. von medienspezifischen Differenzen abhängen. ${ }^{115}$ Nichtsdestotrotz nehmen die letzten erwähnten Zitate Bezug auf einige Eigenschaften von Intermedialität, die eine Einordnung der Komposition erlauben. Es handelt sich in diesem Fall um offene bzw. manifeste Intermedialität: Die Komposition enthält mehr als ein Medium und diese Medien sind distinkt und ,in principle separately ,quotable“". ${ }^{116}$ Da sich die elektronische Komposition an Leverkühns fiktivem Werk orientiert, kann sie als intermediale Transposition eines literarischen Prätextes in eine Medienkombination eingestuft werden, da nicht nur ein Medium in der elektronischen Komposition materiell präsent ist. ${ }^{117}$

\footnotetext{
${ }^{112}$ Zit. im Booklet der CD, S. 8.

${ }^{113}$ Ziolkowski: Leverkühn's Compositions, S. 849.

${ }^{114}$ Zit. in Boehmer: Apocalipsis cum figuris. In: Konrad Boehmer Foundation. $<$ https:// www.kboehmer.nl/compositions/apocalipsis-cum-figuris > (letzter Zugriff: 21.08.2020).

${ }^{115}$ Aussagen über die Qualität eines Werkes bilden bekanntlich den Kern einer Rezension. ${ }^{116}$ Wolf: Musicalized Fiction and Intermediality, S. 243. Vgl. 1.1.5.

${ }^{117}$ Hier wird - im Vergleich zur Komposition von Kurz oder von Ruzicka - immerhin der Begriff der ,intermedialen Transposition“ verwendet, da die Komposition mehrere Mikroformen der Vorlage transferiert und transformiert sowie genau denselben Titel wie Leverkühns fiktives Werk trägt, sodass die Definition ,intermediale Bezugnahme“ unzureichend erscheint. Die Grenzen zwischen einer ,intermedialen Transposition " und einer ,intermedialen Bezugnahme' erweisen sich in der analytischen Praxis manchmal als fließend.
} 
Zudem stellt Boehmers Komposition eine partielles intermediales Produkt dar, das sich auf ein im Roman beschriebenes Werk konzentriert. Resümierend lässt sich sagen, dass Boehmers Komposition etwa durch den Rückgriff auf weitere literarische Texte und Musikstile einen ergänzenden Effekt hat. Sie ist ab und zu in der Lage, die Vorlage zu verstärken, indem etwa die beschriebene Mischung von populären und nicht-populären Musikstilen nicht einfach angedeutet, sondern im musikalischen Text selbst umgesetzt wird. ${ }^{118}$

Wagt man noch einmal den Versuch, Boehmers Komposition im apokalyptischen Diskurs einzuordnen, um sie darin zu verorten, so könnte das Werk als „inverse Warnapokalypse“ bezeichnet werden. Das Erscheinungsjahr der Komposition (1984) spricht ebenfalls für diese Einordnung, da Briese, Faber und Podewski als Beispiel für eine inverse Warnapokalypse u. a. auch den „Warner vor der ,atomaren “ Katastrophe Günther Anders" ${ }^{\text {"119 }}$ nennen. ${ }^{120}$ Charakteristisch für diese Apokalypse ist es, dass „ein unerwünschtes, vernichtendes Ereignis [...] unweigerlich einzutreten“ 121 droht, das aber „durch einen grundlegenden Gesinnungs- und Handlungswandel in der Gegenwart abgewendet werden"122 könnte. In Boehmers Apocalipsis cum figuris könnte durch die Geburt des Kindes das Eintreten eines apokalyptischen Zustandes verhindert werden. Wie in Doktor Faustus und in apokalyptischen Texten der Weimarer Zeit scheint hier kein Gott im Spiel zu sein. Dieser wird in Skrjabins Zitat lediglich erwähnt, es ist aber das menschliche ,aktive[] Handeln“, ${ }^{123}$ welches sich in der Entscheidung zur Fortpflanzung äußert, das zur Erlösung und Transzendenz führt.

Boehmers elektronische Komposition übernimmt die Bildhaftigkeit von Leverkühns Komposition durch die vier Ebenen, lässt sich ebenfalls in den apokalyptischen Diskurs einordnen und interagiert mit populären Musikgattungen.

\footnotetext{
${ }^{118}$ Siehe Gess: Intermedialität reconsidered, S. 141.

${ }^{119}$ Ebd.

${ }^{120}$ Siehe Anders, Günther: Die atomare Drohung. Radikale Überlegungen. München: Beck 1981, 3. durch e. Vorw. erw. Aufl. v. „Endzeit und Zeitende“. Zwar setzt sich „Die atomare Drohung“ aus Schriften von Anders zusammen, die hauptsächlich in den 1960er Jahren veröffentlicht wurden, im Vorwort zur zweiten Auflage von 1981 widmet der Autor sein nach seinen Worten ,leider niemals alt werdende[s]“ Buch jedoch der jungen Generation der "Grünen“ und findet daher eine historisch neue Zielgruppe für seine damaligen Überlegungen. Ebd., S. X. Bemerkenswert ist daneben, dass auch diese Überlegungen im Untertitel als radikal definiert werden. Zur Deklination des apokalyptischen Diskurses im Atomzeitalter siehe auch Derrida: Apokalypse, S. 91-132 (2. Aufsatz des Bandes: „No Apocalypse, not now (full speed ahead, seven missiles, seven missives)“.)

${ }^{121}$ Briese, Faber u. Podewski: Aktualität des Apokalyptischen, S. 21.

${ }^{122}$ Ebd.

${ }^{123}$ Ebd.
} 
Der apokalyptische Diskurs wird dabei um Elemente bereichert, die für die Apokalypsen der 1980er Jahren als typisch gelten. Zudem wird das Stadium der Transzendenz erreicht. Insofern ergänzt die Komposition die literarische Vorlage. Auch Leverkühns Komposition arbeitet mit Umwertungen, da Dissonanz und Tonales darin umgewertet werden: Bei Boehmer wird dieser Aspekt verstärkt und hervorgehoben, denn trotz der scheinbaren Radikalität u. a. der angewandten musikalischen Mittel profiliert sich sowohl in den immer zu hinterfragenden Aussagen der Autorinstanz als auch im Werk selbst eine Umwertung des Weltuntergangs in Erneuerung.

\subsubsection{Konturlosigkeit: die Apocalipsis sine figuris von Karl-Wieland Kurz}

Die Komposition von Karl-Wieland Kurz (*1961), Apocalipsis sine figuris, distanziert sich bereits im Titel von Leverkühns fiktivem Werk. Zwar lässt sie sich anhand des Paratextes sofort auf Thomas Manns Roman zurückführen, das sine figuris jedoch macht gleichzeitig deutlich, dass sich das Werk auf den Roman indirekt bezieht. ${ }^{124}$ Wie bei Boehmer handelt es sich hier ebenfalls um partielle direct or overt intermediality, also offene bzw. manifeste Intermedialität, die erneut der Form der Medienkombination zugeordnet werden kann und sich folglich nicht ausschließlich des Mediums der Musik bedient. Das Wort sine im Titel ist selbstverständlich die Neuheit eines Stückes, in dem die figurae bzw. die Bilder weniger strukturiert eingesetzt werden und in dem Bildhaftigkeit nicht als formbildend konzipiert wird. Nicht zufällig spricht der Autor selbst von „,neutralen Schichten“125 und „Textüberflutungen“, ${ }^{126}$ die den Musikfluss unterbrechen bzw. sogar ,überfluten“. ${ }^{127}$ Während die Schichten Boehmers eine präzise Struktur und

\footnotetext{
${ }^{124}$ Vgl. Scherliess, Volker: Zur Musik in Thomas Manns Roman Doktor Faustus. In: Weber, Hermann (Hrsg.): Literatur, Recht und Musik. Tagung im Nordkolleg Rendsburg vom 16. bis 18. September 2005. Berlin: Berliner Wissenschafts-Verlag 2007, S. 126-154, hier: S. $152 \mathrm{f}$.

${ }^{125}$ Mail vom 08.10.2013 an die Verfasserin.

${ }^{126}$ Ebd.

${ }^{127}$ Ebd. Da wenig Sekundärliteratur zum Stück vorhanden ist, beruhen die Ausführungen dieses Abschnitts fast ausschließlich auf einigen E-Mails (insbesondere auf E-Mails von Kurz an die Verfasserin vom 07.08.2013, 08.10.2013 und 23.10.2013), auf einem persönlichen Gespräch der Verfasserin mit ihm, das in der Schwartzschen Villa in Berlin-Steglitz anlässlich eines Klavierabends (08.12.2013) stattfand, und auf einigen persönlichen nicht veröffentlichten Materialien, die der Komponist der Verfasserin dankenswerterweise zur
} 
Konzeption nachweisen, sind sie bei Kurz neutral und können nicht eindeutig eingeordnet werden. Die Apocalipsis sine figuris ist allerdings nicht gänzlich ohne Bilder: Es werden Kunstwerke gezeigt, das Medium Kunst ist also auch Bestandteil dieses plurimedialen Produktes. Der Unterschied liegt jedoch in der Funktion der benutzten Bilder und Texte: Diese sind neutral, weil sie laut Aussagen des Autors keine verstärkende Funktion haben. Da sie die Musik überfluten, zielen sie auf keine Synthese mit der Musik ab: Sie scheinen als reine, unerwartete Ablenkungen gedacht zu sein, die aber zugleich in der Lage sind, einen Wechsel im Dominanzverhältnis der Medien zu bewirken.

Boehmers Apocalipsis hat die Komposition von Kurz beeinflusst, denn Kurz hat in ihr sogar ein Zitat aus Boehmers Doktorarbeit erwähnt. ${ }^{128}$ Es handelt sich in diesem Fall um eine intermediale Bezugnahme auf den Roman, die auf eine weitere Komposition zu Doktor Faustus Bezug nimmt und daher intramedial als Antwort sowohl auf den Roman als auch auf Boehmers intermediale Transposition aufzufassen ist.

Die Erstfassung der Apocalipsis sine figuris wurde in den Jahren 1985-1987 fertig gestellt und ihre Uraufführung fand 1987 in Frankfurt am Main statt - in der Stadt, in der auch Leverkühns Oratorium uraufgeführt wurde. Die Neufassung mit der Ergänzung des dritten Satzes „Kataleptische Katalekte“ wurde im selben Jahr veröffentlicht und 1988 ebenfalls in Frankfurt uraufgeführt. ${ }^{129}$

Die Besetzung ist in zwei Gruppen gegliedert:

1. Vokalstimmen/Darsteller: vier Sprecher (zwei hohe, zwei tiefe, möglichst männliche Stimmen); vier Sänger: Countertenor, Tenor, Bariton, Bass (alle

Verfügung stellte (z. B. zwei Aufnahmen des Werkes und Erläuterungen). Der Musikverlag Ricordi \& CO, der eine Kopie der Partitur haben sollte, konnte kein Exemplar finden. Ein Beispiel kann man aber auf der Webseite des Komponisten finden: Kurz, Karl Wieland: Apocalipsis sine figuris - Partiturbeispiel. In: Karl-Wieland Kurz. $<$ https://www.karlwiela ndkurz.de/pdf/partituren/Apocalipsis\%20sine\%20figuris.pdf > (letzter Zugriff: 21.08.2020). Die Ausführungen dieses Abschnitts konnten sich daher nicht auf die ganze Partitur stützen. Zur Komposition siehe auch Kurz: Hirn neben Kopf. Vor Apocalipsis, während Apocalipsis und darüber hinaus. In: Musiktheorie 8 (1993) H. 1, S. 37-56.

128 „Denn der Widerstand, den Natur menschlichem Tun leistet, gründet nicht in ihrem subjektiven Willen. Vielmehr ist seine Kraft Zeichen für die unvollkommene Kenntnis, die Menschen über Natur bisher sich aneigneten“. Programmheft Frankfurter Feste 1988, Mensch und Natur, Apocalipsis sine figuris, Alte Oper Frankfurt: 25. September 1988, S. 16.

${ }^{129}$ Vgl. Kurz: Hirn neben Kopf, S. 37. 
Vokalstimmen, über Gesangsanlage); ein Concert-Art-Performer (im Publikum; mit demontiertem Pianino). ${ }^{130}$

2. Orchester: drei Trompeten, drei Hörner, drei Posaunen (Tenor-, Tenor-Bass-, Kontrabassposaunen), drei Tuben (Tenortuba, Bass- bzw. Doppeltuba, Kontrabasstuba); zwei Paukengruppen (möglichst $2 \times 7$ Pauken); Harmonium; Orgel; Celesta (mit elektrischer Verstärkung, oder Sampler); zwei Flügel mit je drei Spielern (vierteltönig gegeneinander verstimmt); zwei Harfen (vierteltönig gegeneinander verstimmt); vier Gitarren (1/2 und 3/4 vierteltönig gegeneinander verstimmt; mit Live-Elektronik); vier Bratschen (eine solistisch; alle mit Pick-up- und Kontaktmikrophonen verstimmt); vier Kontrabässe: der erste in Solostimmung (viersaitig), der zweite Viersaiter in Normalstimmung und die anderen zwei Fünfsaiter (alle Kontrabässe mit Pick-up-und Kontaktmikrophonen verstärkt); Tonbandzuspielung; Mischpult für Klangregie. ${ }^{131}$

Diese detaillierte Beschreibung der Besetzung zeigt, dass das Projekt des Komponisten sehr ambitioniert ist. Noch ambitionierter scheint es, wenn man die riesige Menge der verwendeten Texte betrachtet. Genau wie Adrian Leverkühn reicht Kurz die Offenbarung des Johannes als Quelle eines Werkes über die Apokalypse nicht aus. Für den apokalyptisch-eschatologischen Teil verwendet er Texte, die in der sumerischen Zeit beginnen und sich bis zu den „Berliner Knastblättern“ der 1980er Jahre des 20. Jahrhunderts spannen. ${ }^{132}$ Eine zentrale Rolle spielen die apokryphen und die frühchristlichen religiösen Texte, wie z. B. die Sybillinischen Weissagungen, die Apokalypsen des Thomas und Petrus, Flugblätter und Schriften aus dem Spätmittelalter, teilweise auf Altfranzösisch und Okzitanisch sowie kasuistische Texte. In Anbetracht der Anzahl an verwendeten Texten wäre die Frage berechtigt, ob man den von Gess beschriebenen Effekten von Intermedialität noch einen Effekt hinzufügen sollte, nämlich den Effekt der Revision. ${ }^{133}$ Die Vorlage wird zwar ergänzt und verstärkt, es scheint aber ein Konkurrenzverhältnis zu ihr zu entstehen: Die Distanzierung im Titel durch das sine deutet bereits auf eine revidierende Lektüre von Thomas Manns Roman hin. Zweifellos lässt sich das Stück von Kurz ebenso wie Leverkühns fiktive Komposition als

\footnotetext{
${ }^{130}$ Auf S. 112 wird erläutert, wieso hier nur die männliche Form verwendet wird.

${ }^{131}$ Siehe ebd. und Kurz: Apocalipsis sine figuris - Supplement. In: Karl-Wieland Kurz.<https://www.karlwielandkurz.de/pdf/ApocalipsisSineFiguris.pdf > (letzter Zugriff: 21.08.2020).

${ }^{132}$ Mail vom 08.10.2013.

${ }^{133}$ Siehe Gess: Intermedialität reconsidered, S. 141 u. 1.1.5.
} 
„Resumé aller Verkündigungen des Endes“ (DF: 520) definieren: Der enzyklopädische Charakter bleibt trotz intermedialer Transformation erhalten. Diese riesige Menge von Literatur wird mithilfe eines Montage-Prinzips mit der Musik und mit sich selbst kombiniert: Man erkennt hier das gleiche Prinzip, das in Doktor Faustus identifiziert werden konnte, nämlich die Kombination von Disparatem durch die Montage-Technik. ${ }^{134}$

In der Apocalipsis sine figuris findet man drei Hauptthemen bzw. Hauptideen, die durch das vorher beschriebene Prozedere, jedoch weniger präzise als im Roman organisiert werden, was eine weitere Begründung für das sine figuris darstellt. ${ }^{135}$ Das erste Thema ist selbstverständlich das der Apokalypse, das der Komponist als „bizarre Revue von ,den letzten Dingen“, eine Show vom verschlissenen und beschissenen Ende der Welt" ${ }^{\star 136}$ beschreibt. Das zweite wird als „Galerie der Ideen“"137 bzw. „Zentralfriedhof der abendländischen Ideen“138 bezeichnet und umfasst hauptsächlich Texte aus dem 19. und dem 20. Jahrhundert, u. a. von Freud, Mallarmé, Lautréamont, Bakunin, Cioran und Plechanow. Das dritte Thema ist Adrian Leverkühn gewidmet und versucht, ,einen fiktiven Komponisten so zu porträtieren, als ob er sich bei der kompositorischen Arbeit gerade selbst über die Schulter schaut und sich dabei in einen Bergson'schen unbegrenzten Raum verliert bzw. verströmt". ${ }^{139}$ Dieser Moment lässt sich als metakompositorisch bezeichnen, da über das Komponieren selbst reflektiert wird. Des Weiteren führt die Komposition von Kurz durch die Bezugnahme auf Boehmers Schriften zu einer Reflexion über das „Vertonen“ des Romans von Thomas Mann selbst, was sich als metamedial einstufen lässt. Im Stück herrschen folglich verschiedene Metaebenen: Der Transfer und die Transformation von Mikroformen aus einem Roman, der sich mit der kompositorischen Laufbahn eines Künstlers

\footnotetext{
${ }^{134}$ Siehe dazu Kropfinger „Montage“ und „Composition“ im „Faustus“. Vgl. auch TB2: 19.12.1945, S. 287: „Die Nietzsche'schen Symptome, Medikamente und Speisezettel als Beispiel für das Montage-Prinzip des Buches“.

${ }^{135}$ Die Musikwissenschaftlerin Hanne Stricker erläutert im Programmheft der Uraufführung der zweiten Fassung, was unter der Ergänzung sine figuris zu verstehen ist: „Das sine figuris gibt einen Hinweis auf die scheinbare Konturlosigkeit der Gestalten: es fehlt das Rückgrat. Meint aber auch im musikalischen Sinne, daß die Figurationen fehlen, d. h. die Heterophonie bestimmt weitgehend das Stück. Nur im Rhythmus, der Urgebärde musikalischen Ausdrucks, gibt es noch Verbindung untereinander“. Stricker, Hanne: Dämonenzauber verzerrter Lebensenergien. In: Programmheft Frankfurter Feste, S. 27, hier: S. 27.

${ }^{136}$ Mail vom 08.10.2013.

${ }^{137}$ Ebd.

${ }^{138}$ Ebd.

${ }^{139} \mathrm{Ebd}$
} 
befasst, führt zu Reflexionen sowohl über diesen Transfer als auch über die Inhalte selbst, nämlich den Bereich der Komposition. Um die Rolle von Leverkühn bzw. des im Programmheft genannten „Künstlers“140 zu porträtieren, verwendet der Autor Zitate von berühmten Komponisten des 20. Jahrhunderts, etwa Busoni, Schönberg, Varèse und Skrjabin. Exemplarisch sei hier ein im fünften Teil der Komposition vorkommendes Zitat von Ferruccio Busoni erwähnt: ${ }^{141}$

Nehmen wir es uns doch vor, die Musik ihrem Urwesen zurückzuführen; befreien wir sie von architektonischen, akustischen und ästhetischen Dogmen; lassen wir sie reine Erfindung und Empfindung sein, in Harmonien, in Formen und Klangfarben [...].

Neben vielen anderen Aspekten lässt sich dieses Zitat auf Leverkühns Kompositionstendenzen zurückführen, besonders auf diejenigen, die er mit und nach der Apocalipsis cum figuris verfolgt. Zunächst verfolgt der fiktive Komponist ebenfalls das Ziel, die „Musik ihrem Urwesen zurückzuführen“, indem er sich u. a. auch an Kompositionsstilen vor Bach und Händel orientiert. Zweitens will er ebenso Grenzen und Dogmen sprengen und entwickelt zu diesem Zweck die Zwölftontechnik, die etwa die Grenzen zwischen Harmonik und Melodik, also sozusagen dem Horizontalem und dem Vertikalen der Musik nivelliert. ,[R]eine Erfindung und Empfindung“" ist allerdings die Musik Leverkühns nicht, obwohl in der Weheklag immerhin das Ziel einer Rekonstruktion des Ausdrucks innerhalb einer strengen Form verfolgt wird. ${ }^{142}$ Leverkühns Kompositionskriterien werden durch den Bezug auf Busonis Stellungnahme musiktheoretisch untermauert: Dies verstärkt die Vorlage.

Wie zu Beginn des vorliegenden Unterabschnitts angedeutet, weckt der Titel Apocalipsis sine figuris zum Teil falsche Erwartungen, obwohl hier mit der Polysemie des Wortes gespielt wird und daher $u$. a. auch rhythmische Figurationen in die Gesamtbedeutung des Wortes einbezogen werden. ${ }^{143}$ Das Werk von Kurz besteht nicht nur aus Texten und Musik, sondern auch aus Bildern, etwa der Bildbetrachtung im Teil III 6, in dem die „Offenbarung S. Johannis des Theologen“144 gezeigt wird.

Es gilt, weitere Verknüpfungspunkte mit Boehmers Apocalipsis und mit dem Roman anzusprechen. Zum Beispiel die explizite Stellungnahme des Autors, dass

\footnotetext{
${ }^{140}$ Programmheft, S. 10.

${ }^{141}$ Ebd., S. 15.

${ }^{142}$ Vgl. Kap. 5.

${ }^{143}$ Vgl. Fußnote 135.

${ }^{144}$ Ebd., S. 8 f.
} 
seine Komposition keineswegs den Anspruch erhebt, Leverkühns Oratorium nachzukomponieren, was durch die Änderung des Titels deutlicher als bei Boehmer signalisiert wird. Oder auch die politisch-gesellschaftliche Kritik des Stückes: Diese Kritik wird nicht nur durch die Äußerungen des Autors, er möchte „,die bildungsbürgerliche Haltung von komponierenden Literaturliebhabern zu ihren Schwarmobjekten"145 mitthematisieren, sondern auch durch textuelle Elemente sichtbar. Ein Beispiel dafür wäre das folgende Zitat von Varèse, das am Anfang des Stückes vorkommt: „Die Bourgeoisie heißt Besonnenheit diejenige Feigheit, die sie in ihrer dreckigen Glückseligkeit gelähmt hält". ${ }^{146}$ Sowohl Zeitblom durch seine Erzählungen über die Kridwiß-Treffen als auch Adrian Leverkühn durch sein apokalyptisches Oratorium üben Kritik an der damaligen politischen Stimmung; Boehmer verwendet darüber hinaus, wie in 4.2 .1 bereits angesprochen, Texte von Marx, die Kritik an ökonomischen Machtverhältnissen üben. So kombiniert er die Kritik am Nationalismus und Faschismus mit einer Kritik am Kapitalismus.

Hanne Stricker bezeichnet das Oratorium von Karl-Wieland Kurz als „Dämonenzauber verzerrter Lebensenergien“147 und betont, jene dialektische Ästhetik die in Boehmers Komposition und im apokalyptischen Kapitel von Doktor Faustus sehr wichtig ist - sei in dieser Komposition keine ,formbildende[] Kraft"::148

Er [Karl-Wieland Kurz] rückt bewußt ab von der Dialektik als formbildender Kraft, auch von der Koketterie mit der Ästhetik des Häßlichen und deren Gegensatz, der Schönheit, die als unzuverlässiges Mittel auszuklinken ist. K.-W. Kurz ist entschieden für die Mischung von Disparatem.

Laut dieser Rezension lässt die Komposition von Kurz die Binäroppositionen von Leverkühns apokalyptischem Oratorium, die dort bereits einer Umwertung unterzogen wurden, beiseite und sucht einer poststrukturalistischen Vision entsprechend keine Differenzen zwischen Zeichen mehr: Schönheit und Hässlichkeit bilden einen einzigen Pol. Die Grenzen zwischen den Medien sind fließend, die Schichten werden nicht hierarchisch angeordnet und es herrscht wie in Leverkühns Komposition und bei Boehmer eine Vermischung von Stimmen: Diese Vermischung ist an den verschiedenen Zitaten diverser Autoren aber auch an den

\footnotetext{
${ }^{145}$ Mail vom 08.10.2013.

${ }^{146}$ Programmheft: S. 4.

${ }^{147}$ Stricker: Dämonenzauber, S. 27.

${ }^{148}$ Ebd.
} 
Sprecher- und Sängerstimmen zu erkennen. Auch musikalische Stile werden vermischt: z. B. liest man im vierten Teil die Anweisung: „rhythmisch prägnantes Initial, variiert wiederkehrend; gezacktes, einstimmiges ,Fugato", pointillistisch zerstoben, freie Einschübe, Tonband-Protuberanzen“ ${ }^{149}$ Formen, Strukturen und Systeme werden ebenfalls kombiniert: der erste Teil basiert auf Emergenz, der zweite auf Diskretheit, der dritte auf Fraktalen, der vierte auf einem Modell, der fünfte auf Prozessualität. Nicht nur aufgrund der verwendeten Texte, sondern auch aufgrund dieser Mischung disparater musikalischer Mittel, Stile, Kompositionskriterien und -konzeptionen lässt sich das Oratorium von Kurz dem apokalyptischen Diskurs zuordnen. Aus Gender-Perspektive könnte man außerdem ergänzen, dass die Apokalypse von Kurz stark männlich dominiert ist, da sich diese aus männlichen Stimmen sowie Texten männlicher Autoren zusammensetzt. Es handelt sich daher um eine geschlechtlich markierte Apokalypse: Die Verkündung der bevorstehenden Katastrophe erfolgt eindeutig durch männliche Instanzen.

Sowohl Boehmers Apocalipsis cum figuris als auch die Apocalipsis sine figuris von Kurz stellen eine Erweiterung und Vertiefung des enzyklopädischen Charakters von Manns Kapitel dar, jedoch ist die Wahl der Quellen unterschiedlich und entspricht je anderen Interpretationen des Romans. Beide Komponisten haben es sich nicht zum Ziel gesetzt, wie Adrian Leverkühn zu komponieren und passen den apokalyptischen Diskurs ihrer Epoche an. In beiden Fällen handelt es sich um eine Medienkombination; bezüglich der Komposition von Kurz wäre es aufgrund der fließenden Grenzen zwischen Medien durchaus angebracht, den Terminus der „Medienfusion“150 zu verwenden - obwohl anzumerken ist, dass, wenn die Bilder gezeigt werden, die Musik vorläufig unterbrochen wird und folglich also die Medien nicht wirklich fusioniert werden. Wichtiges Merkmal des Oratoriums von Kurz scheint die Strukturlosigkeit, die Konturlosigkeit, das sine figuris zu sein, was zur formbildenden Kraft der Vertonung unter verschiedenen musikalischen und intermedialen Aspekten wird.

\subsubsection{Die Musikalisierbarkeit von Doktor Faustus: Humphrey Searles Apocalypsis cum figuris}

Die dritte und letzte Komposition, die hier präsentiert wird, ist eine Kantate von Humphrey Searle (Dr. Faustus, im Katalog der British Library The Devil's Jig

\footnotetext{
${ }^{149}$ Programmheft: S. 10.

${ }^{150}$ Rajewsky: Intermedialität, S. 15 .
} 
genannt), die von der BBC Radio 3 am 9. März 1980 übertragen wurde. ${ }^{151}$ Diese Kantate unterscheidet sich wesentlich von den bisher beschriebenen Kompositionen, da sie das Ziel hat, Serenus Zeitbloms Schilderungen von Leverkühns Stücken präzise zu folgen. Der Komponist (1915-1982) selbst schreibt in seinen Memoiren: „I followed Mann’s indications as far as possible“. ${ }^{152}$ Außerdem setzt sich die Kantate nicht nur mit der Apocalipsis cum figuris, sondern auch mit anderen Kompositionen des Romans, z. B. mit der Dr. Fausti Weheklag, auseinander. ${ }^{153}$

Der britische Komponist konnte diese Herausforderung annehmen, weil er in Wien Privatunterricht bei einem der berühmtesten Vertreter*innen der Neuen Musik, Anton Webern, genossen hatte und als erster Komponist gilt, der die 12-Ton-Kompositionstechnik in Großbritannien eingeführt hat. ${ }^{154}$ In seinen Memoiren schreibt er: ${ }^{155}$

I did not particularly want to write a Faust; there have been admirable ones by Berlioz, Schumann, Liszt and Busoni [...]. However when the BBC asked me to write music for a programme about Thomas Mann's Dr. Faustus I could not resist the challenge, especially as Mann's Adrian Leverkühn is very different from the Faust of Goethe, Lenau or the medieval puppet play; he uses the supernatural

${ }^{151}$ Es existiert eine weitere Komposition von Poul Ruders (Corpus cum figuris für großes Ensemble, 1985), die aber nur im Titel und nicht im Inhalt Bezug auf das 34. Kapitel von Doktor Faustus nimmt.

${ }^{152}$ Searle, Humphrey: Chapter 19: Faustus and the Oresteia. In: „Quadrille with a Raven“. <https://www.musicweb-international.com/searle/faustus.htm > (letzter Zugriff: 21.08.2020).

${ }^{153}$ In dieser Studie werden die Apocalypsis und die Lamentation analysiert, da sich diese angesichts des intermedialen Schwerpunktes als besonders aufschlussreich erweisen. Searle hielt außerdem die beiden Stücke für die wichtigsten seiner Faustus-Kantate. Vgl. ebd. u. 5.2.4. Leider fehlt im Fall dieser Komposition sogar ein Teil der Primärliteratur, da das Manuskript, das von der British Library oder von der BBC aufbewahrt werden sollte, nicht mehr gefunden werden konnte. Die vorliegende Analyse basiert hauptsächlich auf der Aufnahme des Stückes, welche die Verfasserin im Oktober 2013 in der British Library hörte, auf einem persönlichen Gespräch mit zwei Schüler*innen und Bekannten von Searle (Herrn Prof. David Sutton-Anderson und Frau Prof. Avril Anderson) und auf den bereits zitierten Memoiren von Humphrey Searle.

${ }^{154}$ Zur Biographie Searles siehe: Mason, Colin, Hugo Cole und David C. F. Wright: Searle, Humphrey. In: Grove Music Online. Zuerst veröffentlicht 20.01.2001, online veröffentlicht 2001.<https://doi.org/10.1093/gmo/9781561592630.article.25279 > (letzter Zugriff: 21.08.2020). Ziolkowski bezeichnet Searle als „British Leverkühn“. Ziolkowski: Leverkühn's Compositions, S. 841.

${ }^{155}$ Searle: Faustus, ebd. 
powers given him by the Devil for the sake of developing himself as a composer rather than on wine, women or power $[\ldots]$.

Sowohl für Boehmer als auch für Searle kommt der Anstoß für die Vertonung von Leverkühns Apocalipsis von außen; beide setzen sich mit Thomas Manns Bearbeitung des Faust-Stoffes auseinander, weil dort die Musik eine zentrale Rolle spielt.

Searle beschreibt sein Projekt nicht als eine Dramatisierung des Romans, sondern als eine Diskussion seiner Hauptideen. So handelt sich auch bei ihm, ähnlich wie bei allen in dieser Studie betrachteten Kompositionen, um kein totales intermediales Produkt, sondern um ein partielles: Trotz des Ziels einer Rekonstruktion der Vorlage ${ }^{156}$ beruht die intermediale Transposition auf einer individuellen Auswahl von zu vertonenden Romankapiteln und -zitaten. Die Kantate ist in eine musikalische Radiosendung eingefügt, in der die Musik manchmal unterbrochen wird, um Passagen aus dem Roman vorzulesen oder wichtige Momente der Handlung zusammenzufassen bzw. zu erklären. Die Auswahl der Texte sowie das Schreiben neuer Texte war Aufgabe des Schriftstellers und Dichters Robert Nye. Am Projekt nahmen auch der Ambrosian Chorus und das New Symphony Orchestra mit den Solostimmen von Wendy Eathorne, Paul Esswood, Brian Burrows und John Gibbs teil. Der Dirigent war Humphrey Searle. Da sowohl das Medium der Musik als auch durch die Moderation das Medium des gesprochenen Textes materiell präsent sind, stellt Searles Komposition, ähnlich wie die von Kurz und Boehmer, eine Form von Medienkombination dar.

Searles Apocalypsis besteht aus zwei Teilen: Der erste ist vom ersten Teil des 34. Kapitels (DF: 511-525), der zweite vom sogenannten "Schluß“ (DF: 538-550) inspiriert. Die Komposition dauert ca. 20 Minuten und die Texte sind hauptsächlich in englischer Sprache. Die Verwendung der englischen Sprache lässt sich nicht als rekonstruktiv bezeichnen. Vielmehr markiert sie einen Unterschied zur Vorlage: Ihre Mikroformen werden nicht nur in das Medium der Musik transferiert, sondern auch sprachlich übersetzt und erhalten somit durch den Sprachwechsel eine nationale Färbung, auch wenn dieser Aspekt bei Searle nicht so ausgeprägt wie bei anderen in dieser Studie behandelten intermedialen Transpositionen, wie z. B. Hagens To Zeitblom, erscheint. ${ }^{157}$

Vor dem Beginn der Musik wird Zeitbloms Beschreibung im ersten Teil des apokalyptischen Kapitels vorgelesen: Der Moderator unterstreicht die Verbindung

\footnotetext{
${ }^{156}$ Vgl. dazu (hier und im weiteren Verlauf der Studie): Eibl, Karl: Kritisch-rationale Literaturwissenschaft. Grundlagen zur erklärenden Literaturgeschichte. München: Fink 1976, S. 68-73.
}

${ }^{157} \mathrm{Vgl}$. 7.2.2. 
der dort geschilderten apokalyptischen Bilder mit Dante Alighieris Commedia, indem er eine literarische Vorlage sowohl der Apocalipsis als auch des Romans selbst (ein Zitat aus Dantes Inferno gehört zu dessen Paratexten) anspricht und unter anderen Quellen privilegiert. ${ }^{158}$ Die Musik beginnt mit glissandi der Pauken und der Posaunen, die sich auf Esmeraldas Thema stützen. Dem ,testis, [...] Zeugen, [...] Erzähler“ (DF: 519) von Searles Apocalypsis wird die Stimme eines Counter-Tenors gegeben. Mit seinen berühmten, rhetorisch als Chiasmus klassifizierbaren Worten „Das Ende kommt, es kommt das Ende“ (ebd.) fängt er an. Später, nachdem das Responsorium seine Worte „unvergeßlich wiederholt“ (ebd.) hat, wird er von dem auch im Roman erwähnten ,,mocking, bleating bassoon in its highest register" 159 begleitet. Der Chor beginnt, begleitet nur von den Pauken, mit Gemurmel, dann singen die Chorsänger*innen immer lauter, während die Musik zu einem Höhepunkt kommt, der durch Fortissimo-Dynamiken und einen massig wirkenden Klang charakterisiert ist. Dieser Höhepunkt führt zu Jeremias Worten: „Wir, wir haben gesündigt/Und sind ungehorsam gewesen“ (DF: 523). Die Chorfuge zeigt in Thomas Manns Roman eine seltsame Form, die Zeitblom so beschreibt:

Ich nenne das Stück eine Fuge, und fugal mutet es an, doch ohne daß ehrsam das Thema wiederholt würde, sondern mit der Entwicklung des Ganzen wird dieses selber entwickelt, so daß ein Stil aufgelöst und gewissermaßen ad absurdum geführt wird, dem der Künstler sich zu unterwerfen scheint, - was nicht ohne Zurückdeutung auf die archaische Fugenform gewisser Canzonen und Ricercaten der vor-Bach'schen Zeit geschieht, in denen das Fugenthema nicht immer eindeutig definiert und festgehalten ist. (DF: $523 \mathrm{f}$.) $)^{160}$

158 „Lo giorno se n'andava e l'aer bruno/toglieva gli animai che sono in terra/dalle fatiche loro, ed io sol uno/m'apparecchiava a sostener la guerra/sì del cammino e sì della pietate,/che ritrarrà la mente che non erra./O Muse, o alto ingegno, or m'aiutate,/o mente che scrivesti ciò ch'io vidi,/qui si parrà la tua nobilitate. DANTE, INFERNO, II. GESANG“. DF: Motto, Herv. i. O. „Der Tag war im Schwinden, und das Dunkel enthob die Lebewesen auf der Erde ihrer Mühen; einzig und allein ich stellte mich darauf ein, den Kampf - mit dem Weg wie mit der Anfechtung - zu bestehen, der nun aus der Erinnerung, bei der es kein Abirren gibt, nachgezeichnet werden soll. Ihr Musen, und du, hohe Begabung, helft mir jetzt! Gedächtnis, das aufgezeichnet hat, was ich sah, hier wird es sich erweisen, wenn du ausgezeichnet bist“". Dante: La commedia, Die göttliche Kömodie, I Inferno - Hölle. Übers. u. komm. v. Hartmut Köhler. Stuttgart: Reclam 2010, 2. Gesang, V. 1-9, S. 27.

${ }^{159}$ Ebd.

${ }^{160}$ Rajewsky zufolge wäre das ein Beispiel für eine Systemerwähnung, eher für eine explizite als für eine evozierende, da fremd- bzw. altermediale Elemente einfach benannt werden und keine „Ähnlichkeitsbeziehung zwischen Elementen und/oder Strukturen des 
Searle versucht, diese Struktur in der Komposition zu realisieren, indem er jede Stimme auf eine unterschiedliche Form seiner Grundreihe (Original, Umkehrung, Krebs, Krebsumkehrung) stützt, obwohl der Rhythmus gleichbleibt. Der resultierende Effekt wird durch ein Fortissimo des ganzen Orchesters unterstützt. Der erste Teil von Searles Apocalypsis endet ,with a picture of the condemned man looking down into the abyss" ${ }^{161}$ eine Anspielung auf Michelangelos Verdammte. ${ }^{162}$

Der zweite Teil fängt „,more light-heartedly“163 mit der Figur der babylonischen Hure an, für die Leverkühn den „graziösesten Koloratur-Sopran“ (DF: 545) vorschreibt. Die „virtuosen Läufe“ (ebd.) der babylonischen Hure „gehen zuweilen mit vollkommen flötenhafter Wirkung in den Orchesterklang ein“" (ebd.), schreibt Zeitblom. Diese Virtuosität realisiert Searle durch spanische Rhythmen und die Sopran-Stimme, die bis zum hohen g gebracht wird. Danach beginnt die Parodie verschiedener Musikstile: „Klänge des französischen Impressionismus, ins Lächerliche gezogen, bürgerliche Salonmusik, Tschaikowsky, Music Hall, die Synkopen und rhythmischen Purzelbäume des Jazz“ (ebd.). Dieser Moment bietet dem britischen Komponisten die Möglichkeit, mit verschiedenen Stilen zu spielen. Robert Nye schrieb passende Nonsenstexte für die „Teufelsgesänge“ (ebd.), die den parodistischen Charakter der Passage unterstreichen.

Danach realisiert Searle das „Pandämonium des Lachens“ (DF: 548), das Zeitblom heftig erschreckt: Genauso erschreckt ihn Leverkühns Neigung zum Lachen. ${ }^{164}$ Das Höllengelächter des Chores fängt leise an, verschiedene und vielfältige Gelächtervarianten sind hier zu erkennen. Es wird bald zu einem ,tornado of sound“, ${ }^{165}$ zum Tutti-Fortissimo des „,sardonischen Gaudium Gehennas“ (DF: 548), das dem Erzähler von Doktor Faustus das Geheimnis der Musik „offenbart“ (DF: 549). Die Wortwahl lässt an die Offenbarung des Johannes denken und das Oratorium Leverkühns zeigt sich hier als eine musikalische Offenbarung des Geheimnisses der Musik statt des Gottes. Dem Höllengelächter folgt der Kinderchor, dieses „Stück kosmischer Sphärenmusik, eisig, klar, gläsern-durchsichtig“

Textes und dem jeweiligen Bezugssystem bzw. entsprechenden Komponenten desselben aufgebaut“ wird. Rajewsky: Intermedialität, S. 89.

${ }^{161}$ Ebd.

${ }^{162}$ Vgl. Buonarroti, Michelangelo: Jüngstes Gericht, 1534-1541. Rom, Vatikanstadt, Sixtinische Kapelle.

${ }^{163}$ Ebd.

${ }^{164}$ Sehr ausführlich ist die Analyse des „Lach-Motivs“ in: Kaiser, Gerhard: „... und sogar eine alberne Ordnung ist immer besser als gar keine." Erzählstrategien in Thomas Manns Doktor Faustus. Stuttgart/Weimar: Metzler 2001, S. 130-134. Vgl. auch Kap. 6.

${ }^{165}$ Ebd. 
(ebd.), das genau wie in Manns Text auf den Noten des Höllengelächters basiert, und der Fähigkeit Leverkühns entspricht, das Gleiche zu verungleichen. ${ }^{166}$ Das Ende beschreibt Searle als,Abgrundmusik' für das ganze Orchester und Orgel. ${ }^{167}$

Im Kontext einer Medienkombination, besonders von Text und Musik, ist die Frage nach dem Verhältnis zwischen den beiden Medien zentral. Der Musikkritiker Malcolm Rayment beschreibt Searles Vorgehen wie folgt: ,When setting a text he is inclined to modesty, submerging his own contribution in the interest of the words“. 168 Dies wird auch von zwei Schüler*innen und Bekannten des Komponisten, Herrn Prof. Sutton-Anderson und Frau Prof. Anderson betont. ${ }^{169}$ Zwar orientiert sich Searles Apocalypsis stark an der Vorlage, übernimmt jedoch zugleich durch die Verwendung der englischen Sprache und vor allem durch den expliziten Einsatz der Zwölftontechnik eine ergänzende Funktion. Programmatisch und durchgehend verwendet Leverkühn Schönbergs Kompositionstechnik lediglich in der Weheklag. Diesbezüglich ergänzt Searles Apocalypsis die Vorlage, indem Dodekaphonie eindeutig und mit der Präzision eines Traktates zumindest bei der Realisierung der seltsamen Fugenform angewandt wird. ${ }^{170}$

Viele Elemente von Leverkühns fiktiver Komposition finden sich in Searles intermedialer Transposition wieder, etwa die babylonische Hure und das KommMotiv: Searles apokalyptischer Diskurs ist daher derselbe Typ wie der der Vorlage; worüber sich die Komposition Gedanken macht, das ist der kompositorische Stil, mit dem sich die verbal music ${ }^{171}$ von Doktor Faustus in das Medium der Musik und in diese spezifische Form von Medienkombination transferieren lässt. Searles Kantate ist insgesamt eine partielle Vertonung des Romans, aber die Vertonung der Apocalipsis cum figuris an sich lässt sich als total einstufen, da kaum ein Element der Vorlage nicht vorkommt. Es handelt sich um direct or overt intermediality; das intermediale Produkt wählt im Vergleich zu den Werken von Boehmer und Kurz Zeitbloms Schilderungen von Leverkühns Oratorium als

${ }^{166}$ Vgl. DF: 549.

${ }^{167}$ Searle: Faustus, ebd.

${ }^{168}$ Rayment, Malcolm: Searle. Avant-Garde or Romantic? In: The Musical Times 105 (Juni 1964) H. 1456, S. 430 ff., hier: S. 430.

${ }^{169}$ Nochmals anlässlich des eingangs des Abschnitts erwähnten Gesprächs der Verfasserin mit ihnen. Vgl. Fußnote 153.

${ }^{170}$ Um mit Sicherheit feststellen zu können, ob das ganze Stück auf die Zwölftontechnik zurückgreift, sollte man die Partitur sichten, was in diesem Fall nicht möglich war.

${ }^{171}$ Der Begriff, der auf Scher zurückzuführen ist (vgl. Einleitung), wird hier und im weiteren Verlauf der Studie im Sinne Werner Wolfs verwendet. Siehe Wolf: „The musicalization of fiction“, S. 133 (insb. Fußnote 3). 
alleinige Vorlage, reflektiert über die Möglichkeiten der intermedialen Transposition, der Musikalisierbarkeit jedes einzelnen Wortes des Textes und versucht, dies umzusetzen.

Im ersten Teil des vorliegenden Kapitels wird gezeigt, wodurch sich die Apokalypse als Redeform auszeichnet und wie sie im Roman musikalisch und politisch dekliniert wird. Diese Verflechtung mit dem Politischen ist sowohl im Roman als auch in der intermedialen Transposition von Konrad Boehmer zentral: Seine Apocalipsis cum figuris reflektiert über Nationalismen und Zivilisationsstadien. Bezüglich der Transposition des 34. Kapitels aus Doktor Faustus ergeben sich einige Kontinuitäten und Differenzen. In die erste Kategorie der Kontinuitäten lässt sich die Beobachtung einordnen, dass alle drei Transpositionen sich nicht nur auf eine apokalyptische Quelle stützen, sondern wie Adrian Leverkühn mehrere Texte kombinieren und zusätzlich auch die Bildhaftigkeit der Apokalypse ins Zentrum stellen, indem sie den Bereich der Kunst einbeziehen. Was die Quellen angeht, so lässt sich zugleich auch eine erste Differenz feststellen: Sowohl Kurz als auch Boehmer ergänzen und passen die Quellen der Vorlage an ihre historische Zeit an. Somit werden Bezüge zum apokalyptischen Diskurs der 1980er Jahre hergestellt, was bei Boehmer besonders deutlich erscheint. Searle, der den Roman so präzise wie möglich transponieren möchte, sieht sich vor die Aufgabe gestellt, einen präzisen Stil für seine Komposition zu wählen und greift auf die Zwölftontechnik zurück, die Leverkühn in der Apocalipsis noch nicht anwendet.

\subsection{Fazit}

Das vorliegende Kapitel bereichert die Rekonstruktion der kompositorischen Rezeptionsgeschichte von Doktor Faustus, indem es eines der Hauptwerke Leverkühns, die Apocalipsis cum figuris, sowie drei intermediale Transpositionen bzw. Bezugnahmen in den Blick nahm. Die Wichtigkeit einer Kontextualisierung des sekundären intermedialen Produktes wurde noch einmal von der Beobachtung bestätigt, dass alle drei Musikwerke in den 1980er Jahren entstanden sind und folglich die apokalyptische Stimmung der letzten Phase des Kalten Krieges in sich tragen.

Im Prozess des Medienvergleichs konnten in diesem Kapitel weitere Effekte von Intermedialität und speziell des Medienwechsels beobachtet werden: Erstens die Ergänzung der Vorlage, etwa bei Boehmer und Kurz durch die Erweiterung der Quellen, die bei der Beschreibung von Leverkühns Komposition erwähnt werden, was zugleich für ein adaptives Textverständnis spricht. Zweitens eine eher rekonstruktive Haltung gegenüber der Vorlage, z. B. bei Searle, der Zeitbloms 
Beschreibung der Apocalipsis, also jenes intermediale telling hörbar zu machen beabsichtigt. Mediengrenzen erschienen bei diesem Versuch zugleich fließend und scharf gezogen: Nicht alles kann in Musik gesetzt werden und ausgerechnet das Medium der Schrift erwies sich aus diesem Blickwinkel als vage. Searle löst dieses Problem ebenfalls durch die Ergänzung der Vorlage mittels der Anwendung der Zwölftontechnik, die im Roman erst in der Weheklag verwendet wird. Mit dieser Komposition, die es in dieser Untersuchung zudem ermöglicht, die Frage nach der Erreichung eines transzendentalen Stadiums im apokalyptischen Diskurs zu beantworten, beschäftigt sich das nächste Kapitel.

Open Access Dieses Kapitel wird unter der Creative Commons Namensnennung - Nicht kommerziell - Keine Bearbeitung 4.0 International Lizenz (http://creativecommons.org/lic enses/by-nc-nd/4.0/deed.de) veröffentlicht, welche die nicht-kommerzielle Nutzung, Vervielfältigung, Verbreitung und Wiedergabe in jeglichem Medium und Format erlaubt, sofern Sie den/die ursprünglichen Autor(en) und die Quelle ordnungsgemäß nennen, einen Link zur Creative Commons Lizenz beifügen und angeben, ob Änderungen vorgenommen wurden. Die Lizenz gibt Ihnen nicht das Recht, bearbeitete oder sonst wie umgestaltete Fassungen dieses Werkes zu verbreiten oder öffentlich wiederzugeben.

Die in diesem Kapitel enthaltenen Bilder und sonstiges Drittmaterial unterliegen ebenfalls der genannten Creative Commons Lizenz, sofern sich aus der Abbildungslegende nichts anderes ergibt. Sofern das betreffende Material nicht unter der genannten Creative Commons Lizenz steht und die betreffende Handlung nicht nach gesetzlichen Vorschriften erlaubt ist, ist auch für die oben aufgeführten nicht-kommerziellen Weiterverwendungen des Materials die Einwilligung des jeweiligen Rechteinhabers einzuholen.

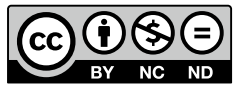

